\title{
Paracelsus als wissenschaftlicher Schriftsteller
}

\author{
Ist die deutsche Sachprosa eine Lehnbildung der lateinischen \\ Schriftkultur? [1994]
}

\author{
et bene dicit, quia sermo \\ divinus est suavis et planus, \\ non altus et superbus sicut \\ Virgilii et poetarum \\ Benvenuto da Imola
}

Die Ausbildung einer allgemeinen deutschen Schriftsprache ist das herausragende Thema der Sprachgeschichten der Neuzeit, aber die Frage, die hier am weitesten führt, ist bisher geradezu stiefmütterlich behandelt worden:

Ist die deutsche Schriftsprache aus der mündlichen Sprachkultur hervorgewachsen, oder entstand sie im Bruch mit ihr, als Lehnbildung der lateinischen Schriftkultur? - Nach meinem Eindruck spricht sehr viel für die zweite Möglichkeit.

Wir Germanisten sind als Sprachhistoriker weit mehr Erben der romantischen Nationalphilologien als die Mediävisten, die sich in Gelehrten wie Schwietering und Ohly schon vor fünfzig Jahren aus dieser Tradition gelöst haben, während wir die Herkunft unserer Sprache aus dem lateinischen Mittelalter keineswegs genügend ins Auge fassen. Die lingua europaea universalis et durabilis ad posteritatem, ${ }^{1}$ die noch Leibniz erhalten wissen wollte, jene ,europäische Universalsprache, die eine dauerhafte Überlieferung garantiert‘, bildet in der Neuzeit auf fast allen in Betracht kommenden Gebieten den Hintergrund sich allmählich verselbständigender landessprachlicher Schriftkulturen (besonders lehrreich zu diesem

1 Gottfried Wilhelm Leibniz: Kurzes wohlgemeintes Bedenken vom Abgang der Studien und wie denenselben zu helfen. Denkschrift 1711 (zitiert nach Leibniz 1916a: XXIf.).

\footnotetext{
Anmerkung: Wertvolle Anregungen und Hinweise verdanke ich Herrn Michael Kuhn, der bei meiner Paracelsus-Vorlesung des Wintersemesters 91/92 hilfreich assistiert hat und seine Magisterarbeit („Der zeichen art ist betrüglich“) über Sprache und Signatur bei Paracelsus geschrieben hat (1991), ebenso der Untersuchung des lateinischen Hintergrunds einer chirurgischen Vorlesung Hohenheims in Basel, die Frau Carolin Holtmeier zum Thema ihrer wissenschaftlichen Arbeit gemacht hat (1992), Seminaren über Paracelsus seit dem Wintersemester 86/87, zunächst mit dem Medizinhistoriker Heinz Schott. - Die meisten Einblicke in das schwer zugängliche Bergwerk Paracelsus verdanke ich meiner Frau.
}

2 Open Access. (C) Uwe Pörksen, publiziert von De Gruyter. (c) BY-NC-ND Dieses Werk ist lizenziert unter der Creative Commons Attribution-NonCommercial-NoDerivatives 4.0 Lizenz. 
Thema Eggers 1963-1977, Bd.1: 50ff., 217f. u. ö.). Latein war die Sprache der Kirche und weltlicher Herrschaft gewesen und blieb es auf lange Zeit, es dominierte selbst während der „Stauferzeit“ in der Poesie und seit Ende des 15. Jahrhunderts auch im Bereich der Rechtstraditionen. Als Sprache der Wissensüberlieferung, der Gelehrsamkeit, war es vermutlich der höchste Zacken in dem steilen lateinischen Gebirgspanorama.

Von ihm sind auch die naturkundlichen und heilkundlichen Texte des hohen und ausgehenden Mittelalters abhängig, sie gehören fast immer - man entschuldige die Bildkonsequenz - zu seinem Geröllfeld. Ich gestehe, zunächst oft überrascht gewesen $\mathrm{zu}$ sein: Man glaubt, einen mittelhochdeutschen Originalton zu hören, einen volkssprachlichen, der auf mündlicher Überlieferung beruht, und dann ist es doch wieder eine Übersetzung oder Kompilation aus dem Lateinischen. Noch Dürers (1525) Übersetzung von Ellipse als ,ei-lini` in der für Malerknaben gedachten Underweisung der messung hat ihr Äquivalent im lateinischen Oval.

Vermutlich ist es die Blickrichtung unserer nationalen Sprachgeschichtsschreibung, die diesen Hintergrund eher zurücktreten lässt, weil man nach vorne schaut auf das unterstellte Ziel einer einheitlichen Nationalsprache (vgl. den anregenden Aufsatz Knoop 1987). Der auf diese Zukunft ausgerichtete teleologische Prozess erscheint dann vor allem als ein Sichherauswinden, als Emanzipation. Schaut man in entgegengesetzter Blickrichtung, blickt man also zurück, so wird das Umgekehrte wahrscheinlicher. Die deutsche wissenschaftliche Prosa hat sich nicht auf der Grundlage einer eigenen untergründigen mündlichen Kultur herausgebildet, sondern als Lehnprägung der lateinischen Schriftkultur. Erst in letzter Zeit wird dies bewusster. Die neue Sprachgeschichte von Peter von Polenz (1991), Deutsch in der frühbürgerlichen Zeit, genau, fundiert, differenziert wie immer, hat da einige neue Markierungen gesetzt und geht weit über Eggers hinaus (zahlreiche Hinweise enthält auch das quellenreiche Werk von Wells 1990).

Gilt das Gesagte auch von Paracelsus? Wie nimmt er sich aus vor dem Hintergrund des lateinischen Massivs? Ist er von ihm abhängig oder eher ein mündlicher Autor, einer, der auf mündlichen Traditionen fußt und deren Sprechweise aufgenommen hat?

Die Schreibart scheint irgendwo zwischen holpriger Mundartlichkeit, rhetorischem Kunstgriff und lateinischer Schreibunterlage angesiedelt. Wo liegt ihre Einheit? Ist Paracelsus tatsächlich der neben Luther hervorragende Schriftsteller der frühen Neuzeit, der auf dem Gebiet der Sachprosa den Sprachhorizont seiner Epoche am weitesten aufsprengt, am umfänglichsten und gründlichsten bereichert, eine zum Vorbild gewordene Prosa erarbeitet hat? Oder widerspricht eine solche Frage dem Stil der Epoche, den Intentionen Hohenheims und ist im Grunde anachronistisch? 
Er ist als Autor noch umstrittener als es der Theologe, Naturphilosoph und Arzt gewesen ist; bemerkenswerterweise beginnt ein Streit um den Schriftsteller überhaupt erst im 20. Jahrhundert. Joachim Telle hat in seinem Aufsatz Die Schreibart des Paracelsus im Urteil deutscher Fachschriftsteller des 16. und 17. Jahrhunderts (1981) zunächst Urteile aus unsrer Epoche zusammengestellt.

Da gibt es das Wort von dem „großen Prosaschriftsteller“ seines Zeitalters, von der „Körnigkeit und Plastizität“ seines Stils und seiner „Sprachmächtigkeit“, von „wundervoller Sprachgewalt“ und „bildhaft kernigem“ Deutsch. Einmal ist die Rede von dem „schwerzüngigen Genie“, „das mehr aussagen will, als für seine Zeit schon wortreif ist“. Diese Stimmen sind in der Minderzahl, und es hat den Anschein, als drückten sie sich nicht besonders genau aus.

Dem stehen andere gegenüber, die sich ihre Mühsal mit Theophrasts Sprache eingestehen und die Ursache in dem Autor suchen. Sie sprechen von ,altertümlichen“ Zügen, einer „schwerfälligen“, „unbeholfenen Sprache“, „ermüdender Breite und langsamem Gedankenfortschritt“, „Undeutlichkeit“, „Dunkel“ und „Ungeschicklichkeit des Wortes“. Paracelsus liege „stets im Kampfe mit der Sprache“, einer, der, „was er sagen wollte, [...] nicht denkend durchzuformen vermochte“. Ein „Wust“, bar jeder literarischen Eleganz, „kraus, bald umständlich, bald abrupt“, sei diese Diktion und auch seine Terminologie oft „willkürlich“, „magisch-mystisch“, selten erreiche sie „volle begriffliche Prägnanz“. Unter den Kritikern sind die Namen Gundolf, Hankamer, Schipperges, Lippmann, Wollgast und Löther, Goldammer (Nachweis der Zitate bei Telle 1981: 78ff.). Es sei die Sprache, schreibt Kurt Goldammer (1953: 30), die auf dem Wege zu einem vertieften Werkverständnis „oft ein entscheidendes Hindernis“ bilde, und Max Wehrli (1980: 1025) meint aus diesem Grunde, Paracelsus sei Literarhistorikern „Gegenstand einer gewissen Verlegenheit“. Gisela von Boehm-Bezing (1966), die in ihrer detaillierten Untersuchung zu Stil und Syntax bei Paracelsus zu einem sehr kritischen Ergebnis gelangt, wird als Kronzeugin hervorgehoben.

Auch als Fachschriftsteller im engeren Sinn genießt Paracelsus nach der Überzeugung Joachim Telles (1979) in unserem Jahrhundert einen falschen Ruf. Er war nicht der kometenhafte Einzelgänger und maßgebliche Bahnbrecher.

Angesichts des ansehnlichen Raumes, den das Deutsche im Bereich der medizinischen, pharmazeutischen und alchemischen Fachliteratur seit dem Spätmittelalter dem Latein abgewonnen hatte, zerbröckelt allmählich die alteingeschliffene Anschauung, ein kühner medizinischer Schriftsteller Paracelsus habe - im Unterschied zum Verfasser theologischer Schriften - literarisch noch ungeebnetes Terrain betreten und ohne Vorgaben im schreibsprachlich Ungebahnten als erster medizinische Sachgebiete in deutscher Sprache behandelt. Die Menge deutschsprachiger Handschriften und Drucke medizinisch-naturkundlichen Inhalts aus vorparacelsischer und Paracelsischer Zeit nötigt dazu, statt von Hohenheims 
,Einführung‘ der deutschen Sprache in die Medizinliteratur von einer ,Fortführung älteren Sprachverhaltens zu sprechen [...]. (Telle 1979: 81)

Das Urteil der Deutschen Wortgeschichte, Paracelsus habe die Sprache der Medizin, Alchemie und Pharmazie „entscheidend beeinflußt, ja die deutsche Sprache für diese Wissenszweige recht eigentlich erst ausdrucksfähig gemacht“" (Rosenfeld 1974: 502), die Auffassung von Dietlinde Goltz (1972: 340f.), seine Schriften hätten neben dem Werk Luthers „entscheidend zum Entstehen einer deutschen Hochsprache bei[getragen], mit der es möglich wurde, auch wissenschaftliche Themen zu behandeln“, erscheint ihm als äußerst zweifelhaft.

Diese Auffassung stützte sich vor allem auf die Dissertation von Karl-Heinz Weimann (1963), Paracelsus und der deutsche Wortschatz, die, nur in Auszügen veröffentlicht, die einzige Untersuchung auf diesem Gebiet darstellt. Weimann (1963: 374, 367) ist der Ansicht, Paracelsus habe den deutschen Wortschatz in „ungewöhnlicher“, „unerhörter“ Weise bereichert, man müsse ihn ,als Glied, fast als Anfangsglied, einer gemeineuropäischen Bewegung sehen“. Er nennt zahlreiche Wörter, die sich nach seiner Überzeugung durch Paracelsus im Deutschen eingebürgert haben, assimilierte Fremdwörter wie Alkohol und Gas, Homunculus und Undine, metallisch, Natron, Signatur, Sublimierung, Tinktur, aber auch Neubildungen aus dem Erbwortschatz wie Amtsträger und Eiweiß, Erkältung und Frauenkrankheit. - Telle erwidert, Gerhard Eis und Herwig Buntz hätten nachgewiesen, dass manches, was Weimann Hohenheim zuschrieb, vor ihm existent sei, nicht nur als Wort, sondern auch in dem neuartigen Gebrauch, dass also seine Verdienste um unseren Wortschatz geringer seien (vgl. Telle 1981: 82, der Bezug nimmt auf Eis 1965b und Buntz 1973).

Man wird sagen müssen, dass beide Fragen noch offen sind: Solange das Paracelsus-Wörterbuch, das vor Jahrzehnten geplant worden und dann eines ungeklärten Todes gestorben ist, nicht wieder zum Leben erweckt und erarbeitet wird, können wir uns über Art und Umfang, in denen Paracelsus den deutschen Wortschatz erweitert hat, nur vorläufig äußern und müssen uns an Einzelbeispiele und an die vorhandenen Glossare halten, z.B. an das vorzügliche Wörterbuch, das Hans Kayser seiner Auswahl beigegeben hat (vgl. Paraceslsus 1924). Die Glossare sprechen m. E. für Weimanns Annahmen. ${ }^{2}$ - Solange wir zweitens keinen deutschen Olschki (1919/1922/1927), keine Geschichte der neuzeitlichen naturkundlichen und medizinischen Sachliteratur haben, können wir die Bedeu-

2 Vgl. die Glossare des 16. Jahrhunderts von Dorneus \& Bodenstein 1981, Toxites 1574 und die des 20. Jahrhunderts von Kayser in Paracelsus 1924: 434-486, Strebel in Paracelsus 1949: VIII, 459504, Jacobi in Paracelsus 1942: 259-278, Weimann in Paracelsus 1955: 277-287 sowie Weimann 1951. 
tung Hohenheims für die Ausbildung unserer Sachprosa, seine Nachwirkung nicht wirklich abschätzen.

Wie wichtig sind aber überhaupt diese Fragen? Treffen sie Hohenheim in seiner Zeit an, in seinem Wirkungswillen, oder gehören sie nicht vielleicht eher in den Rahmen nationaler Geschichtsschreibung und Sprachgeschichtsschreibung, zugespitzt gesagt, in das Gebiet der Herstellung einer Ahnenreihe nationaler Gipsbüsten? Sind sie nicht von sekundärem Interesse und überdies anachronistisch?

Telle (1981) untersucht im Hauptteil seines Aufsatzes die Frage, wieweit der Autor Paracelsus von den Fachschriftstellern des 16. und 17. Jahrhunderts genannt und als Vorbild anerkannt worden sei, nachdem ihm oder weil ihm ,von Historikern des 20. Jahrhunderts das Verdienst zuerkannt [wurde], den Aufstieg der deutschen Landessprache zum anerkannten Medium der Medizin, Pharmazie und Alchemie maßgeblich gefördert zu haben“, und stellt abschließend fest:

Erwartungswidrig mußten wir aber bei einer Umschau im Bezirk deutscher Druckschriften chirurgischen, leibärztlichen und alchemisch-chemiatrischen Inhalts feststellen, daß Paracelsus als sprachliches Vorbild ungenannt bleibt und die frühneuzeitlichen Urteile über seine Schreibart fast ausnahmslos ungünstig ausfielen (Telle 1981: 99).

Das Ziel, durch vorbildhafte Spracharbeit zur Ausbildung einer Nationalsprache beizutragen, hat Hohenheim nicht verfolgt, wie Leibniz es entwirft im Anschluss an Schottel in seiner Ermahnung an die Deutschen, ihren Verstand und ihre Sprache besser zu üben“ von 1682/83, wo er die Ausarbeitung ,annehmlicher Kernschriften “ fordert, eines Kanons der Sachprosa in deutscher Sprache, „damit der Lauf der Barbarei gehemmt und, die in den Tag hinein schreiben, beschämt werden mögen“ (Leibniz 1983: 77). Hohenheims Intention war andersartig, sie ging auf die Sache, er hat sich, wie einer der von Telle zitierten Autoren meint,

die fundamenta der Aristotelischen Philosophey sampt den gantzen Galenischen secten, die Artzney belangende umbzustoßen underfangen, in maßen, daß im fahl er den Clavam und Herculis kolben inn henden gehabt hette, Namlich einen zirlichen stilum zuschreiben (dessen er sich dann wie man sihet nicht geachtet) so wer er zweifelsone genugsam sterck gewesen, zuvertilgen biß an Herculis säulen die gantze Galenische und Arabische Medicin, und seine säulen mit mehreren Triumph vil weiter hinauß zu pflantzen, dann Galenus, Avicenna, und all andere so seidher des Großen Hippocratis gewesen, und zu verdunckeln mit seiner Sonnen jr großes lieht. (Zitiert nach Telle 1981: 99, dort nach Gilly 1977: 91)

Der ,zirliche stilus‘, das Humanistenideal einer rhetorisch geschmückten Sprache, wird hier lediglich als das erwünschte Werkzeug betrachtet, um den Sturz Galens und aller Säulen der traditionellen Medizin zu erreichen. An diesem Werkzeug 
gebrach es ihm in der Tat; die von Telle aufgefundenen Urteile stehen durchweg in der Tradition der Rhetorik und des Vorwurfs der Dunkelheit, wie ihn, vergleichbar, Gottfried von Straßburg gegenüber Wolfram von Eschenbach erhoben hat (vgl. Tristan, 4638-4690). Im Sinn der klassischen Stiltradition schrieb er „schlecht“; das sagt aber nur bedingt etwas über die Qualität seiner Sprache und gar nichts über seine Bedeutung für die Geschichte der Sachprosa. Theoretisch könnte Paracelsus, gerade weil er dies Stilideal des ,ornatus‘ nicht verkörperte, weil sein Ehrgeiz nicht in der Sprache lag, einen besonders weitreichenden Einfluss gehabt haben.

Wenn wir von dem Schriftsteller Paracelsus sprechen, müssen wir im übrigen bedenken, dass seine Epoche ihm nicht erlaubt hat - oder war es das, was ihm als sein apostolisches Amt erschien, sein ruheloses Wanderleben oder auch nur sein widerborstiges Naturell? -, sich im Dialog mit Druckstätten und einem Publikum zum Schriftsteller heranzubilden. Es gab hier keine Wechselwirkung zwischen Autor und Leserschaft, er nahm nicht Profil und Form an, weil er einen Verleger vor Augen hatte und ein bestimmtes Publikum ansprechen konnte. Eine Rückwirkung der neuen Druckmedien und des Marktes, wie sie Michael Giesecke (1991) zum Thema gemacht hat, lässt sich bei diesem Autor besonders schwer beobachten. Zu seinen Lebzeiten erschienen von ihm 1529 und 1530 bei Peypus in Nürnberg Syphilisschriften, in denen er ein beliebtes Heilmittel, das von den Fuggern aus Südamerika importierte Holz Guayak, in Zweifel zog und sich ein Druckverbot einhandelte, 1536 dann in Augsburg die Große Wundartzney, die in Fachkreisen fast wirkungslos geblieben $\mathrm{zu}$ sein scheint. Auch sie erregte am Druckort Anstoß. Der Grußbrief Talhausers, eines befreundeten Augsburger Arztes, den Paracelsus in die erste Auflage aufgenommen hatte, musste auf Verlangen der Augsburger Ärzteschaft im Neudruck von 1537 draußen vor bleiben, Talhauser 1538 die Stadt verlassen. Die übrigen während seines 48jährigen Lebens gedruckten Sachen sind einige wenige Gelegenheitsarbeiten, eine Basler Vorlesungseinladung, Prognostikationen, Deutungen von Kometenerscheinungen und anderen Himmelszeichen, eine schmale Schrift aus dem Jahr 1535 Von des Bades Pfäfers Tugenden, Kräften und Wirkung, Ursprung und Herkommen, Regiment und Ordnung.

Er war kein Schriftsteller, der wie Luther mit Hilfe der Drucktechnik zur Institution geworden wäre, oder wie nach ihm Galileo Galilei eine literarische Strategie entwickelt hätte, sondern ein vermutlich meist nächtlich rastloser Skribent, der den Gastgebern Dokumente seiner geistigen Stationen hinterließ, dessen Freunde seine Manuskripte sammelten. Manches davon ist mehrfach geschrieben, liegt in drei oder vier Fassungen vor, einiges ist sorgfältig durchgearbeitet und wie aus einem Guss, die Septem Defensiones zum Beispiel oder der Liber de nymphis, der Labyrinthus medicorum errantium oder auch eine 
große Schrift wie die Astronomia Magna. Vieles trägt das Gepräge des Vorläufigen, manches des Flüchtigen und Unkonzentrierten. Er diktierte meist. Was von ihm übrig ist, ist weniger ein Werk, als eine Hinterlassenschaft. Die von Sudhoff 1922-1933 herausgegebenen medizinischen, naturwissenschaftlichen und naturphilosophischen Schriften umfassen 14 Bände, die von Kurt Goldammer betreuten Theologischen und religionsphilosophischen Schriften liegen bis jetzt in acht nicht weniger starken Bänden vor und sind ebenfalls auf vierzehn geplant (vgl. Paracelsus 1955-1995). Von diesen 28 Bänden ist dem Umfang nach etwa einer zu seinen Lebzeiten erschienen.

Unter diesen Umständen ist es nicht ohne weiteres möglich, Paracelsus für die neuen Spielregeln der „,,Verschriftlichung des Lebens“ in der frühen Neuzeit“ (vgl. Giesecke 1992) anzuführen. ${ }^{3}$ Gewiss hat er hervorragenden Anteil an der „Umstrukturierung der Wissensbestände und Erfahrungsregistratur in der Fachprosa“, die Michael Giesecke für diese Epoche beschreibt. Es gibt kaum ein Gebiet, durch das er nicht hindurchgegangen, auf dem er nicht seine eigenartigen Spuren hinterlassen hat: Mariologie und Steinleiden, Pest- und Syphilistraktate, Wundarznei, Pharmazie, Ethik des Arztberufs, Bergbau und Bergbaukrankheit, Bäder, Astrologisches, Pflanzen, Steine, Metalle, Kosmogonie, Psalmenauslegung und eine neue Theorie der Sozialordnung - aufgeworfen in einem 48jährigen Leben. Auf welche Weise aber fügte er seine Schriften ein in eine kommunikative Situation, schloss sich an an vorhandene Titulatur und Darstellungsmuster, formulierte er den Umriss eines Publikums? Die Titel bedürften einer gründlichen Untersuchung, z.B. die nur an Hand der Manuskripte zu klärende Frage, wie weit Paracelsus oder Huser oder Sudhoff ihr Urheber ist, ob sie eher originell und abweichend sind (was häufig der Fall zu sein scheint) oder in die neue Typologie der „Fachbücher“ eingepasst. Wie sehr wechselte er, je nach Gegenstand und erhofftem Publikum, Darstellungstyp und Wortschatz? Seine Schriften stellen sehr unterschiedliche Ansprüche. Wen wollten sie ansprechen? Die zahlreichen Praefationes wären eine ergiebige Quelle.

Beobachten wir die Mittel jener ,Dekontextualisierung، und ,Verständigungssicherung‘, von denen Giesecke (1992: 90) spricht? Ist die Darstellung, auf Grund der neuen situationsenthobenen Konstellation des Buchdrucks, im Hinblick auf das Publikum veranschaulicht, verbegrifflicht und verallgemeinert, wie von ihm postuliert (vgl. Giesecke 1992: 93ff.)? Wie weit reichte die „Sozialisation“ dieses fast nur zu einem imaginären Publikum redenden Verfassers? Es wäre eine eigene

3 Die Habilitationsschrift Gieseckes 1991 und die Studien 1992 sind für unser Fach überhaupt erst fruchtbar zu machen und können hier nur gestreift werden. Der konkrete Fall Paracelsus fügt sich auch unter dem Blickwinkel Gieseckes m. E. nur schwer der allgemeinen Tendenz. - Siehe auch das bahnbrechende Werk von Eisenstein 1979. 
Studie erforderlich. Wenn Michael Giesecke (1992: 100) Hohenheims Verteidigung seiner neuen Krankheitsbegriffe und Krankheitsnamen als Ausdruck des neuzeitlichen Zeitbegriffs und des mit ihm vermachten Wissensfortschritts, eines beschleunigten „Wissensumschlags“ interpretiert, so ignoriert er den ganz andersartigen hier zugrundeliegenden Gedanken, dass Krankheiten für Paracelsus mit den Gestirnen korrespondieren und dass der Wandel der Gestirnskonstellationen notwendig ihren Wandel nach sich zieht. Wenn er ihm „das Programm einer Verknüpfung des theoretischen Wissens der lateinischen Litteratustradition mit dem muttersprachlichen Wissen der Handwerkertradition" unterstellt (Giesecke 1992: 102), so ist das zumindest missverständlich, da Paracelsus sich ja zur Schrifttradition entschieden querstellt, und wenn er, im Anschluss an ein Zitat aus der Großen Wundartzney, die ,gemeinen' Laien zu den Adressaten Hohenheims erklärt, so ist auch das so nicht richtig: er meinte hier die Wundärzte. Die Zitate sind aus dem Zusammenhang gerissen. Wie deutlich ihm andererseits der Zusammenhang zwischen Zielgruppe und Sprache war, verrät eine Stelle, auf die mich Michael Kuhn hinweist: „nun hab ich zu Basel vom caduco gelesen, aber nach dem und dieselbe zeit die auditores warent: anderst und anderst wer gelesen worden, so ich ander und ander auditores gehabt het“. ${ }^{4}$

In einem Fall haben wir ein klares Bild. Die kleine auf den 31. August 1535 datierte und zu Hohenheims Lebzeiten gedruckte Schrift Vonn dem Bad Pfeffers in Oberschwytz gelegen“, in ihrer Sperrigkeit und Dunkelheit zunächst kaum zugänglich, ist gerade nicht „dekontextualisiert“, sondern wird erst aus ihrer Situationsgebundenheit verständlich. Die Übersetzerin und letzte Herausgeberin Gunhild Pörksen vergleicht diese untypische Bäderschrift einem Telegramm:

\begin{abstract}
Ihm geht es offenbar nicht darum, die Kranken, die von weither kommen, über die lokalen Besonderheiten von Pfäfers in Kenntnis zu setzen, sondern er unternimmt es, den Ort der Quelle im Zusammenhang des Makrokosmos zu bestimmen und von dorther ihre Kräfte zu verstehen. Hier zeigt sich zugleich ein Hinweis auf das mögliche ,Zielpublikum' dieser Schrift: sie ist nicht nur, wie aus der Widmung hervorgeht, an Johann Jakob Russinger, Abt zu Pfäfers, gerichtet, mit dem Paracelsus mutmaßlich schon zehn Jahre zuvor in Gespräch und Austausch gewesen ist und der im Sommer 1535 sein Patient war, sondern sie ist nach meinem Eindruck deutlich für einige Menschen bestimmt, die dem ,medizinischen Personal ${ }^{*}$ im Bad angehört haben. (Pörksen, G. 1993: 138)
\end{abstract}

4 Paracelsus: Von den hinfallenden Siechtagen der Mutter (S I, 8: 320). Hier und im Folgenden zitiere ich, wenn nicht anders vermerkt, nach der Ausgabe Paracelsus 1922-33, abgekürzt S I (plus Band- und Seitenangabe). 
Die kosmologische Unterweisung in der Vorrede, eine Abbreviatur von mehr als fünfzig Seiten der um diese Zeit entstehenden Astronomia Magna (1537/38), scheint so unmenschlich schwierig auf Grund dieser Verkürzung. Der naturtheologische Eingang der Schrift wird am ehesten begreiflich, wenn er als ein ,aidememoire“ für schon Unterrichtete niedergeschrieben ist, und auch der praktische Teil dieser Handreichung für das Badepersonal ist so etwas wie das „Protokoll“ eines erfahrenen und geübten Fragers, der für das Kloster und die ,Verwalter` des Bades festhielt, was er erkundet hat:

Wie wirkt die Quelle bei Schußwunden? Wie bei Pfeilwunden? Wie, wenn Pfeiltrümmer in die Wunde hineingeheilt sind? Wie bei eingekapselten Kugelfragmenten [...]. Vieles, was nachher in der Großen Wundarznei (1537/38) dargestellt wird, wird hier stichwortartig angesprochen. ${ }^{5}$

Schließlich ist auch das Glossar, das er der Schrift angehängt hat, im Unterschied etwa zu einem gelehrteren, anspruchsvolleren, welches er in die Entwürfe zur Astronomia Magna einfügte, ein Indiz dafür, dass das ungelehrte Personal des Bades sein erstes Zielpublikum gewesen ist, jene, die seine Verordnungen zu erweitern verstehen, „dan die teglich erfarnheit übertrift die ratschleg der buecher“ (S I, 9: 655):

Das kleine Lexikon, die Uslegung der latinischen synonyma so in den vergangnen capiteln gedruckt sind worden machte nicht allzu viel Sinn, wenn es an den hochgebildeten Abt gerichtet wäre. Es muß für Leute gedacht sein, die kein Latein verstehen! Paracelsus gibt immer die einfachste, prägnanteste Erklärung, in einem kurzen Satz, z.B. Materia ist ein stuck, das man in die hand nimpt. Operatio ist die würkung, so die natur vollbringen mag. Flamula sind hanenfüß. Aperiren ist öffnen. (Pörksen, G. 1993: 143)

Ob die Schrift für den Druck gemeint war? Paracelsus hat in den beiden größeren Werken, die zu seinen Lebzeiten gedruckt wurden, den Syphilistraktaten und der Großen Wundartzney, sich weniger an die Situation gebunden und deutlicher auf die Spielregeln der neuen Schriftlichkeit acht gehabt, aber es bedürfte einlässlicher Untersuchungen, um zu sehen, wie weit. Als Schriftsteller sieht und bewegt er sich auch hier vorrangig in einem anderen Spannungsfeld als dem der Drucktechnik, von dem übrigen unveröffentlichten Schrifttum zu schweigen.

Die ungezählten Blätter sind bisher weder als Sprachwerk noch in ihrem Sachgehalt erschlossen, und ich fürchte, wir werden die heikle Frage, ob er ins Pantheon gehört, auch hier nicht lösen können. Wir können vielleicht das Span-

5 Zitate aus Gunhild Pörksens Einleitung in Paracelsus 1993: 15, 16f.; vgl. auch Pörksen, G. 1993: 138. 
nungsfeld verdeutlichen, in dem er sich bewegt, und diesem den Umriss des Schriftstellers Paracelsus einzuzeichnen versuchen.

Ich schlage vor, dass wir uns dem Autor von vier Seiten nähern:

1. Paracelsus und die 1. Person Singular;

2. Nominalismus und Sprachkritik;

3. der lateinisch-deutsche Fachwerkstil und die Lehnprägung durch das Lateinische;

4. Sermo humilis.

\section{Paracelsus und die 1. Person Singular}

Das ,Rollenwort‘ Ich, von dem Brinkmann (1971: 743f.) in seiner Grammatik spricht, gewinnt bei Paracelsus eine in der Sachprosa bis dahin unbekannte Dimension; fast könnte man sagen, es rückt in den Mittelpunkt. Hohenheims Wahlspruch, der auf den beiden bekannten Porträtstichen von Augustin Hirschvogel überliefert ist, Alterius non sit qui Suus esse potest, ,Es sei nicht eines Andern, wer sein Eigner sein kann', entspricht durchaus dem Tonfall seiner Schriften.

Schauen wir zurück, so gibt es bei Hildegard von Bingen am Ende des 12. Jahrhunderts erstaunliche individuelle Selbstzeugnisse, in denen sie von ihrer visionären Gabe oder von der Gebrechlichkeit ihres Körpers spricht, aber dies Selbstbild ist nicht Teil ihrer naturkundlichen und heilkundlichen Prosa. Und bei Konrad von Megenberg, in seinem deutschsprachigen Buch der Natur von 1350, lesen wir zwar gelegentlich, ,ich, Megenberger, glaub das nicht‘, erfahren wir seine Zweifel an irgendwelchen Fabeleien oder daran, ob seine Vorlage ein Albertus Magnus sei, aber das Ich wird nicht Fluchtpunkt seiner Darstellung der Naturdinge. ${ }^{6}$

Bei Paracelsus gewinnt die Art, in der er ich sagt, eine andere Qualität. In dem Augenblick, wo eine fast zweitausendjährige Überlieferung fraglich wird, wo ein Ich sich ihr gegenüber und sie auf den Prüfstand stellt, wird der Prüfstein selbst unvermeidlich zum Thema. Es sind wechselnde Anlässe, aber fast immer ist es die Position der Verteidigung, aus der heraus er von sich spricht, der Rechtfertigung. Das Ich wird zur Grundlage und zum Argument.

In seiner ersten sicher datierbaren Schrift, dem am 15. August 1524 in Salzburg verfassten Libellus De Virgine Sancta Theotoca, einem Flugschriftchen, das

6 Siehe z.B. die Quellenauswahl in der Ausgabe der Schriften Hildegards von Johannes Bühler, Hildegard von Bingen 1922: 18-37, und Konrad von Megenberg 1962: 27, 236, 427 u. ö. 
die himmlische Herkunft Mariens verteidigt, erklärt er, er greife zur Feder, weil er in einer Disputation versagt habe und überhaupt nicht in der Lage sei, vor höheren Herrschaften Rede und Antwort zu stehen. Drei Doktoren der Theologie hatten ihn an dem Ort, wo er seine colloquia gab, vermutlich in einer Schenke, aufgesucht, ihn offenbar in Verwirrung gestürzt, und ihn jetzt freundlich eingeladen, am Erzbischöflichen Hof zur Disputation $\mathrm{zu}$ erscheinen. Er lehnt ab. Nachdem er schon vor ihnen in Stummheit erstarrt sei, wie viel mehr würde er vor dem Fürsten erzittern. Der kleine, am Brustkorb verwachsene Mann mit dem wuchtigen, früh kahlen Schädel war ein Stotterer.

Darumb, daß ich etwas rwlich abgezogen bin, ist das die mehrer ursach, daß ich dreyen euch gelehrten mit meiner stamleten zungen nicht folgen mag, als ihr dann mich erkennt von Jugend auff, daß ich die allemal stamlet getragen hab [...].

Er ist nicht nur seiner Zunge nicht Herr, sondern sieht sich auch als Underdog, „schenden“ geboren, wenn Sudhoff und Biegger den Text richtig entziffern, und ist einem Gedankenandrang ausgesetzt, der ihn nicht die eingeschlagene Richtung einhalten lässt, weil Assoziationen und Einreden ihm dazwischengeraten, so dass es anders herauskommt als beabsichtigt. Vielleicht spiegelt sein Stil gelegentlich diese Behinderung, was möglich ist, weil er diktierte. Es hat etwas Stoßweises, oft Abruptes, ein Hinterstzuvörderst, was Satzbau und Gedankenordnung manchmal undurchsichtig werden lässt. Auch die eben paraphrasierte Stelle wird am ehesten verständlich, wenn man sie mündlich vorträgt und den Satz durch Intonation gliedert:

\begin{abstract}
Aber so ich meiner Zungen und zufliegenden rede nicht gewaltig, und mein selbst gewiß bin $\mathrm{zu}$ eröffnen, das so mein hertz begehrt, ungeschickligkeit ein ursach ist, das, dz Ich schenden [?] erzogen bin, die ander ursach ist: die dritt, daß mir im reden die ungezämten gegenred und die abweg so da mitlauffen, meiner zungen mein geist nehmen, berauben mir meines hertzen gemütt, und eröffnen ein anders dann ich im sinn hab. [...] Aber so mein stamlet zung nicht kan das verantworten, gehört denn dasselbige meiner rechten hand zu mit gefaßter Feder auszubreiten [...]. (Sudhoff 1899: 296; vgl. die Transkription bei Biegger 1990: 33)
\end{abstract}

Er schreibt, weil er schüchtern ist, fasst sich und gibt sich schreibend eine Fassung, ängstlich, ein Stotterer, der unter Gedankenflucht leidet und sich in der mündlichen Rede keine Zügel aufzuerlegen weiß. Schreiben wird zur Kompensation eines Mangels, es tritt für ihn ein.

Ein realistischer, um nicht zu sagen, naturalistischer Zug kommt hier in seine Selbstdarstellung, der sich später in seinen Septem Defensiones von 1537/38 erhärtet, wo er in der sechsten, „zu entschuldigen sein wunderliche weis und zornige art“, seine Schroffheit durch seine Herkunft erklärt. Er sei nicht in Seide 
und mit Feigen aufgewachsen, sondern unter Tannenzapfen. „mein fürnemen ist, mit dem maul nichts gewinnen, alein mit den werken“ (S I, 11: 151f.). Es fällt andererseits schwer, hier ganz den Gedanken an jene Überlieferung fernzuhalten, der zufolge Moses „nicht wohl beredt“ war, sondern „eine schwere Sprache und eine schwere Zunge hatte“ (2. Mose 4, 10), an das Motiv der Unbeholfenheit des berufenen Knechtes.

In einer zweiten Flugschrift, De septem punctis idolatriae cristianae von Anfang 1525 (Paracelsus 1955-1995, III: 2-5), sehen wir, wie er sich als Antitypus kirchlicher Amtsträger verfasst, ein Sendbote der Wahrheit und geißelnder Prophet, der zur Gottesdienstzeit in Tavernen, Krügen, Wirtshäusern wider das unnütze Kirchengehen, Beten und Fasten auftritt und die Vorwürfe, die gegen ihn gerichtet werden, in sein Bild übernimmt. Der ironische Kontrast war sein Element: die Wahrheit aus dem Mund eines Stammlers vor den Bauern der Taverne. Man zieh ihn des Suffs, nannte ihn einen ,Winkelprediger', ein, wie wir aus den Wiedertäuferakten wissen, dem Scharfrichter vorarbeitendes Etikett. Im Mai 1525 verließ er fluchtartig die Stadt.

Zur Erweiterung der Facetten dieses Autoren-Ichs wähle ich das Buch Paragranum, das 1529/30 in verschiedenen Fassungen entstand, nachdem Paracelsus seine Position in Basel verloren und sich in Nürnberg durch seine Syphilisschriften ein Druckverbot eingehandelt hatte. Ein doppelt gereizter, hochempfindlicher Autor.

werd ich geursacht, hie das buch Paragranum zu schreiben, darin ich tractir den grund aus dem ich schreib, den grund on den kein arzt wachsen mag, darin ich mich sovil entplös, das endlich mein herz meniglich sol geoffenbart werden [...]. (S I, 8: 51)

dan niemants schreiet, dan der verwunt wird; niemand wird verwunt, dan der empfintlich ist; niemand ist empfintlich, dan der zergenglich ist und nicht bleiblich. sie dieselbigen schreien, dan ir kunst ist zerbrüchlich und tötlich; [...] also sind sie tötlich, darumb schreien sie wider mich. (S I, 8: 53)

Er selbst schreit am deutlichsten. Es gibt in der Sachliteratur vermutlich kein vergleichbares Dokument der Herzensentblößung. Er zitiert die Feindbilder, die man sich von ihm macht, die Namen, die ihm seit Basel anhaften, ,Cacophrastus“, der ,Waldesel von Einsiedeln‘. Man hat ihn des Plagiats geziehen, seiner Heilerfolge wegen der Teufelsbündelei, ihn um Honorare geprellt und deshalb verlacht. „mit was spot habt ir mich ausplasimirt, ich sei Lutherus medicorum, mit der auslegung, ich sei haeresiarcha? ich bin Theophrastus und mer als die, den ir mich vergleichent; [...]“ (S I, 8: 62f.).

Er ist zum Ketzer geworden, ruft Widerstand hervor und scheidet die Geister. Der Autor des Paragranum sieht sich als Beginn eines neuen Äons. „wie dunkt euch, so secta Theophrasti triumphiren wird und ir werden in mein philosophei 
müssen und euern Plinium Cacoplinium heißen und euerm Aristotelem Cacoaristotelem [...]?“( (S I, 8: 56f.)

Seit Basel imitiert er die Reformatorenrolle und zielt auf vollständige Konfrontation. Den Namen ,Lutherus medicorum‘ hat er sich selbst zuzuschreiben; selbst noch Luthers Verbrennen der Bannbulle und der kanonischen Rechtsbücher hat er nachgeahmt, indem er in Basel ein medizinisches Kompendium ins Johannisfeuer warf, um, wie er im Rückblick sagt, seine Monarchei einzuleiten, d.h. in seinem Sprachgebrauch seine Epoche. „nun schauet, wes die monarchei sei, euer oder mein?“ (S I, 8: 58). Das dualistische, polare Gegenüber, hier Wahrheit - dort Lüge, hier Ich - dort Ihr, hier Christus - dort der Leibhaftige, bestimmt den Selbstentwurf des Autors, dessen Naturbild eigentlich gar nichts Manichäisches hat und der im Paragranum ein anziehendes, tiefgründiges Bild des neuen Arztes und einer posthumoralen Medizin entwirft. Lag hier eine durch das Zeitklima bedingte Verirrung vor? Ein absurdes Selbstbewusstsein spricht aus der Schrift; die einen haben aus solchen Gründen einen Prahlhans in ihm gesehen, die anderen einen Außerordentlichen, der wie Luther oder Calvin die vocatio extraordinaria für sich in Anspruch nehmen konnte.

[...] ich nicht euch nach, ir mir nach. mir nach Avicenna, Galene, Rasis, Montagnana, Mesue etc., mir nach und nit ich euch nach, ir von Paris, ir von Mompelier, ir von Schwaben, ir von Meißen, ir von Cöln, ir von Wien und was an der Donau und Rheinstrom ligt, ir insuln im meer, du Italia, du Dalmatia, du Sarmatia, du Athenis, du Griech, du Arabs, du Israelita, mir nach und ich nicht euch nach. eurer wird keiner im hindersten winkel bleiben, an den nicht die hunde seichen werden. ich werd monarcha und mein wird die monarchei sein, und ich füre die monarchei und gürte euch euere lenden. wie gefelt euch Cacophrastus? diesen dreck mußt ir essen. (S I, 8: 56).

Es geht ein bisschen mit ihm durch, und am Ende setzt er, wie oft an vergleichbaren Stellen, und wie es übrigens auch Luther in der Schrift An den Christlichen Adel deutscher Nation getan hat, die Narrenkappe auf. Seine Glatze wisse mehr als Avicenna und alle Hohen Schulen (S I, 8: 39), meint er gelegentlich, er stilisiert sich als Vagant, Kuckuck, Gauch, und spielt auf seine dürftige, im Nacken sich krümmende Tonsur an, das ,gauchhaar‘; in Thüringen hießen diese widerspenstigen Haare „zankhärchen“.

ich sage euch, mein gauchhar im genick weiß mer dan ir und alle eure scribenten und meine schuchrinken [Schuhschnallen] seind gelerter dan euer Galenus und Avicenna und mein bart hat mer erfaren dann alle euer hohe schulen. ich wil die stund greifen, das euch die seu im kat müssen umbziehen: wie gefelt euch der peregrinus? wie gefelt euch der waldesel von Einsidlen? brecht herfür, was steckt in euch, könnet ir disputiren? (S I, 8: 65).

Ein Shakespearescher Narr, der die Welt auf den Kopf stellt. 
Schon in Basel hat Paracelsus mit einer Autorität gebrochen, die jahrhundertelang bestanden hat: mit der an einen Kanon lateinisch gefasster Überlieferung gehefteten Wahrheitsvermutung. In seiner berühmten Intimatio vom 5. Juni 1527, dem als Flugblatt veröffentlichen Vorlesungsprogramm (vgl. S I, 4: 1-4), verlagert er den Wahrheitsbeweis weg von der Autorität der Alten und schriftlicher Überlieferung auf die selbsteigene Erarbeitung der Natur der Dinge.

Damit wird das Ich des Wissenschaftlers, sein Verstand und seine Arbeit, seine Erfahrung an und mit der Natur zum Ausgangspunkt, die Rechenschaft darüber zur Legitimation. Die Rechenschaft wird im Extremfall zur wissenschaftlichen Autobiographie.

Bei Paracelsus finden wir ihre ersten Ansätze. In der Großen Wundartzney von 1536 gibt es Passagen, in denen er glaubwürdig macht, dass er dies Werk auf Kopf, Auge und Ohr gegründet habe. ${ }^{7}$ Er ist nicht mehr nur, als der umstrittene einsame Prophet, die Identifikationsfigur, sondern auch die Induktionsfigur des Lesers. „Ich hab ie und ie mit großem aufsehen fleißiger arbeit mich geflissen zu erfaren den grunt in der arznei, ob sie doch müg ein kunst geheißen werden oder sein oder nicht, oder was doch in ir seie. dan darzu hat mich bewegt vilerlei ursach [...]“, beginnt die Vorrede (S I, 10: 19). Nachdem alles ungewiss sei oder Schwindel, die Kranken einer hilflosen ,kunst“ ausgeliefert, die alten Schriften voller Torheit und bei den in Seide und goldenen Ringen gehenden Ärzten der Fürstenhöfe nichts zu holen, habe er, bei der vollständigen Ungewissheit der medizinischen Kunst, erwogen, ihr den Rücken zu kehren, bei sich die Schuld gesucht, die hohen Schulen bei den Deutschen, Italienern, Franzosen aufgesucht (er sagt ,erfaren`), aber nicht allein in ihrer Gelehrsamkeit sich umgetan,

sonder weiter gwandert gen Granaten, gen Lizabon, durch Hispanien, durch Engeland, durch den Mark, durch Prüchsen, durch Litau [...] auch sonst andere lender nit not zu erzälen, und in allen den enden und orten fleißig und empsig nachgefragt, erforschung gehapt, gewisser und erfarner warhaften künsten der arznei. nicht alein bei den doctoren, sondern auch bei den scherern, badern, gelerten erzten, weibern, schwarzkünstlern so sich des pflegen, bei den alchimisten, bei den klöstern, bei edlen und unedlen, bei den gescheiden und einfeltigen. hab aber so ganz grüntlich nicht mügen erfaren, gewiß zu sein, es sei in was krankheit es wöll. hab im vil nachgedacht [...]. (S I, 10: 19f.)

7 In anderen Schriften betont Paracelsus neben der visuellen besonders die taktile Wahrnehmung; ,alein was die augen sehen und was die finger tasten, dasselbig lernet den theoricum medicum“ (S I, 11: 24). Ähnlich im Opus Paramirum (S I, 9: 177) u. ö. Vgl. dazu auch Giesecke 1991: $691 \mathrm{f}$. 
Es ist erstaunlich, dass Goethe diese Vorrede nicht vor der Abfassung des FaustMonologs gelesen hat. Die Reichsunmittelbarkeit des Ich, seines Erkenntnisweges, ist die gleiche.

ich hab gesehen zu griechischen Weißenburg ein Walachen der gab nicht mer dan ein trunk zu trinken [...] ich hab auch in Crabaten gesehen von einem Zigeiner, der nam ein saft von einem kraut [...] ich hab auch gesehen zu Stockhalma in Denmarck ein wunttrank bei einer edlen frauen [...] ich hab auch gesehen ein mal einen teufelbeschwörer [...],

heißt es auf einer Seite des laufenden Textes (S I, 10: 95f.). Das Ich, sein Erfahren und Prüfen, wird zur Sonde der Wahrheit.

Am Ende legt er Rechenschaft ab über seine Ausbildung, es ist der gleiche Duktus wie zu Beginn:

von kintheit auf hab ich die ding getriben und von guten underrichtern gelernet, die in der adepta philosophia die ergründesten warent und den künsten mechtig nach gründeten. erstlich Wilhelmus von Hohenheim, meinen vatter, der mich nie verlassen hat, demnach und mit sampt im ein große zal, die nit wol zu nennen ist [...] (S 1, 10: 354).

Er nennt einige.

Das stärkste Beispiel für dies Verfahren, zugleich, wenn man so will, die erste Autobiographie eines wissenschaftlichen Autors in unsrer Sprache, sind Hohenheims Septem Defensiones. Verantwortung über etliche Verunglimpfungen seiner Mißgönner von 1537/38.

,Die viert defension, von wegen meines lantfarens', vertieft den Gedanken, dass sich hier ein Ich zum Instrument der Welterfahrung gemacht hat. Er spricht vor allem von seinem Auge. Man kennt den Holzschnitt Albrecht Dürers, auf dem das festgestellte Auge eines Zeichners durch einen schachbrettartig gerasterten Schirm auf eine Figur schaut, eine nackte Liegende, und er das Wahrgenommene, sein Auge am Peilstab, exakt auf ein schachbrettartig gerastertes Blatt überträgt, das vor ihm liegt. ${ }^{8}$ Das Bild gilt als Emblem der Epoche, in der die Egozentralperspektive beginnt und das Subjekt zu dem wird, was das Wort wörtlich bedeutet: das zugrunde Liegende.

Paracelsus verteidigt seine Nichtsesshaftigkeit, die nicht nur freiwillig war, denn kurz vor dem Desaster in Basel hatte er sich in Straßburg bei der Zunft der ,Luzerne' das Bürgerrecht erkauft, - er verantwortet diese anstößige Zigeunerei damit, dass die ,künste‘ über die ganze Welt ausgeteilt seien und zusammen-

8 Vgl. die schöne Interpretation Der Zeichner des liegenden Weibes in der Einleitung zu Kutschmann 1986: 21-27. 
geklaubt werden müssten, und indem er den Gedanken, dass das Auge das Organ sei, um sie sich anzueignen, durch alle Fächer des Wissens durchführt.

Wie mag hinder dem ofen ein guter cosmographus wachsen oder ein geographus? gibt nicht das gesicht den augen ein rechten grunt? so laß nun den grund besteten. (S I, 11:142)

was ist das on das gesicht bezeugt mag werden? hat sich got nicht selbst mit augen zu sehen geben, und stellet uns zu einer zeugnuß das unsere augen in gesehen haben? wie wolt dan ein kunst oder anders sich der augen zeugnuß entschlahen? (S I, 11: 142)

Er nennt die Säulen der Medizin, die er im Paragranum errichtet hat, Astronomie und Kunde der unteren Erde und Alchemie; alle haben im Auge die Quelle des Wissens, den Zeugen, das Beweisinstrument. Der Schluss ist berühmt:

also acht ich, das ich bisher mein wandern bilich verbracht hab, mir ein lob und kein schant zu sein. dan das wil ich bezeugen mit der natur: der sie durchforschen wil, der muß mit den füßen ire bücher treten, die geschrift wird erforschet durch ire buchstaben, die natur aber durch lant zu lant: als oft ein lant als oft ein blat. also ist codex naturae, also muß man ire bletter umbkeren. (S I, 11: 145f.)

Es ist ein Epochenwechsel, der in solchen Sätzen zum Ausdruck kommt. Das überlieferte Bild vom Buch der Natur erhält eine gründliche Wendung. ${ }^{9}$ Kein buchgeleitetes Wissen mehr, keines, das durch die Brille kanonischer Schriftüberlieferung hinausschaut, sondern das Ich, die erste Person Singular, als Erwanderer der über die Welt ausgestreuten Wahrheit. Als Körper mit Auge, Händen und Füßen, als kümmerliches, defektes Gefäß, Mittler, Ort der Wahrheit und Leidender am Widerstand der Menge, Identifikationsfigur und Induktionsfigur, Richter über die Erkenntnis.

Indem es Ich sagt, lädt es den Hörer ein, sich an seine Stelle zu setzen, so wie es umgekehrt sich in dessen Position versetzt. Eine ganz abstrakt gemeinte Stelle aus der Grammatik Brinkmanns (1971: 743f.) über die Pronomina Ich und Du scheint hier aufschlussreich:

Diese Pronomina sind dadurch gekennzeichnet, daß sie Rollen bezeichnen, die von den Teilnehmern eines Gesprächs wechselnd besetzt werden: Der Sprecher ist potentieller Hörer, der Hörer potentieller Sprecher, und sie sind eben nur insofern Sprecher und Hörer, als sie zugleich ,Anwärter‘ auf die andere Rolle sind. Diese Pronomina stehen in einem Verhältnis der unablässigen wechselseitigen Substitution.

Mit der Betonung dieser Rollenwörter kommt ein demokratischer Zug in die Wissenschaft; die moderne Naturwissenschaft ist grundsätzlich demokratisch:

9 Vgl. die Bemerkungen zu dieser „empirischen Wanderschaft“ bei Blumenberg 1989: 69. 
Jedes Ich hat hier gleiches Recht. - Bei Paracelsus wird allerdings dieser demokratische Zug konterkariert durch das Bewusstsein, auf Grund besonderer Berufung der Wahrheit teilhaftig zu sein und sich unter sie zu beugen: ein Zwiespalt der Epoche.

In Hohenheims Entwurf werden bereits alle Konturen des wissenschaftlichen Schriftstellers als jenes maßgeblichen Weltinterpreten erkennbar, dem wir später in Gestalten wie Galileo Galilei oder Thomasius, Linné oder Haeckel begegnen.

\section{Nominalismus und Sprachkritik}

Der Naturforscher der Neuzeit ist in der Regel Nominalist, er sucht sich eine Vorstellung von den Dingen zu machen, indem er von der Wahrnehmung des einzelnen ausgeht und darauf seine Begriffe aufbaut. Zugleich besteht für ihn eine prinzipielle Kluft zwischen Wörtern und Dingen, er neigt zu der Ansicht, die schließlich de Saussure als das Prinzip der Beliebigkeit des sprachlichen Zeichens formuliert hat: Es gibt keine notwendige, natürliche Beziehung zwischen Wort und Ding. Der Wahrheitsgehalt einer Aussage ist nicht an eine fertige, vorgegebene sprachliche Form gebunden, sondern grundsätzlich von ihr $\mathrm{zu}$ trennen, insofern sprachfrei: ${ }^{10}$ Das scholastische voces significant res mediantibus conceptibus, ,Lautzeichen bedeuten Dinge auf dem Weg über die mit ihnen verbundenen Begriffe‘ (vgl. Gauger 1972b: 114f.), heißt für ihn, dass in den Wörtern auch falsche Begriffe überliefert sein können. Gerade die sprechenden, durchsichtigen, motivierten Ausdrücke müssen Gegenstand kritischer Untersuchung werden und mit ihnen jene traditionsreiche Sprachtheorie, die Namen als Zeugnisse uralter Weisheit verehrt und ihnen die Vorstellung von den Dingen entnimmt. Der neuzeitliche Forscher sucht sich den Dingen direkt gegenüberzustellen und lehnt es ab, sich von der überlieferten Sprache die Begriffe vorschreiben zu lassen, auch, oder gerade dann, wenn es um die Heiligen Sprachen Hebräisch, Griechisch, Latein geht. Denn mit ihnen vor allem war die umgekehrte Theorie verbunden, die Sprache selbst beherberge die Wahrheit, und das Wesen der Dinge sei aus den Namen zu erschließen.

Paracelsus steht, überwiegend, auf der Seite der Neueren. Einen Sonderstatus der drei Sakralsprachen lehnt er rundweg ab; nur beim Hebräischen, der Sprache des Paradieses, gibt es in einer nicht sicher paracelsischen Schrift, De natura rerum, eine Ausnahme: „das aus hebraischer sprach auch die rechten namen

10 Eine wertvolle Anregung verdanke ich hier der Wissenschaftlichen Arbeit von Heidrun Fröschle 1992. Zum Thema vgl. z.B. Apel 1975. 
herfließen und erfunden werden“ (S I, 11: 397f.; vgl. Wegener 1988: 123). Kritik an überlieferten Ausdrücken, die Schaffung eigener neuer Termini gehört bei ihm zum fortwährenden Geschäft. Er hat nicht zuletzt durch seine neuartige Sprache Anstoß erregt. Als er in Basel lehrte, im Sommer 1527, hing eines Morgens ein gegen ihn gerichtetes Schmähgedicht an den Kirchentüren der Stadt, Manes Galeni adversus Theophrastum, potius Cacophrastum, in dem es hieß:

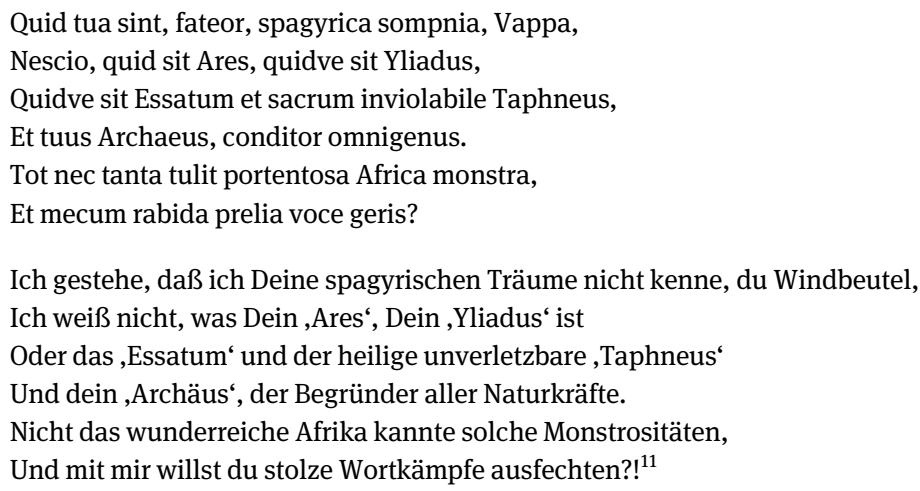

Einige von den hier angefochtenen Ausdrücken, Yliadus, Archaeus, hat er zu Schlüsselbegriffen seiner Naturphilosophie entwickelt; es war von heute her gesehen in der Tat ein neuer Kontinent, der sich hier ankündigte.

Über den Sprachbegriff Hohenheims ließe sich ein Buch schreiben, das sich allerdings nicht auf eine zusammenhängende Schrift stützen könnte, sondern aus vielen verstreuten Äußerungen in seinem Werk zusammenzutragen wäre - so wie man auch die Facetten der Ich-Aussagen beträchtlich erweitern könnte. Vor Linné wüsste ich keinen Naturexperten, der so gründlich über Sprache und Terminologie nachgedacht hat. Ich stütze mich im Folgenden auf eine kleine Quellenauswahl, ${ }^{12}$ die frühe Schrift Eilff Tractat oder Buecher Vom Ursprung und ursachen Der Wassersucht / Der Farbsuchten / Deß Schlags etc. (evtl. 1520), die Syphilisschriften, Von der französischen Krankheit. Drei Bücher (1529/30), verschiedene Fassungen des Paragranum (1529/30), die zweite Defension, ,betreffend die neuen krankheiten und nomina des vorgemeldten doctoris Theophrasti` (1537/38), und halte skizzenhaft fest:

11 Text und Übersetzung nach Sudhoff 1936: 38ff.

12 Hier danke ich Michael Kuhn 1994; 1996, der an einer Dissertation ,De nomine et vocabulo Sprachbegriff und Krankheitsnamen bei Paracelsus arbeitet, für wertvolle Hinweise. 
I Er verwirft, ohne sich in der Praxis ganz danach zu richten, die dem Mittelalter so geläufige Denkform der figura etymologica (vgl. z.B. Klinck 1970), die von den Bestandteilen des Namens einer Sache auf die Sache schließt: „Vom namen so die wassersucht hat, es sei zu latein, zu griechisch, arabisch, chaldeisch, laß dich nit bekümmern in ir etymologia, dan da spielen miteinander die sprachen / und scherzen, wie die kazen mit den meusen; es ist on nuz" (S I, 1: 3). Man solle sich um die tradierten Namen nicht kümmern, sondern nach der Ursache der Krankheit fahnden, der ,causa efficienc', dem ,rechten grund', und danach den Namen geben.

II Er verwirft die kanonische Autorität arabischer oder griechischer Nomenklaturen, weil Krankheiten seiner Überzeugung nach, wie auch die zugehörigen Heilmittel, regionalen Charakter haben. Im diametralen Gegensatz zur späteren Naturwissenschaft meint er, einem jeden Land oder Landstrich wachse seine Krankheit, sie sei kein Universale, ihr Name daher nicht über weite geographische Distanzen übertragbar. Die Erhebung arabischer oder griechischer Benennungen zur verbindlichen klassischen Norm erscheint als eine Form von Sprachimperialismus. „got hat die Kriechen nit zu irdischen göttern gesezt oder der arznei Evangelisten zu sein, wie sie in Granaten, in Portugall etc. sol gehalten werden. sie haben sich aber Rom vergleicht, [...].“ (S I, 7: 130)

III Eine solche ,Impostur', ein solcher Betrug, scheint ihm auch deshalb nicht erlaubt, weil er Krankheiten für historische Phänomene hält. Ihre Namen und Theorien und die damit korrespondierenden Heilmittel sind auch über historische Distanzen nicht übertragbar - eine uns nicht mehr ganz so fremde Auffassung. Krankheiten sind der Zeit unterworfen, sie wandeln sich mit dem Altern der Welt, mit ihren Äonen oder Monarchien. Diese Welt ist nicht die zeitlose, physikalisch ewige Natur der naturwissenschaftlichen Epoche nach ihm, sondern ein geschichtliches Wesen, das sich gegen sein Alter immer weiter von der Jugend entfernt. Erde und Wasser, der gesamte Makrokosmos, Luft und Feuer, dies an erster Stelle, der Sternenhimmel haben entscheidenden Einfluss auf die Entstehung von Krankheiten, und da die Himmel ihre Sterne wechseln, das Firmament seinen Frühling, Sommer und Herbst hat, ändert sich auch seine Operation, seine Wirkung und sein Werk, modeln sich buchstäblich die Ur-Sachen der Krankheiten um. Er schreibt in der ,anderen Defension':

Nun ist der Himmel auch ein Kind gewest, hat auch ein Anfang gehabt und ist in das End prädestiniert als der Mensch und mit dem Tod umgeben und verfasset. So sich nun ein jedlich Ding in das Alter ändert, so ändern sich auch dieselbigen Werk. So nun Änderung der Werk da sein, was nützet mich dann die Ruten der jungen Kind? Darum red ich von der jetzigen Monarchien von wegen des Alters des Firmaments und der Element. Weiter auch so ist vorhanden ein solche Menge des Volks und solche Vermischung unter ihnen durcheinander mit allem Wandel der Menschen in fleischlichen Begierden, als vor nie gewesen, 
solang die Welt gestanden ist. Daraus folgt nun ein solche pressura gentium, dergleichen auch nie gewesen ist. Also folgt aus dem auch ein Arznei, die vor nie gewesen ist. Darum mag sich der Arzt des nicht behelfen, der da spricht, ich behilf mich der Bücher, die vor zweitausend Jahren geschrieben sind. Es sind nimmer mehr dieselbigen causae [...].“ (Paracelsus 1991: 15)

IV Der Wandel der Welt, die Entdeckung neuer Phänomene macht neue Namen erforderlich. Er spricht vom Selbstmord - Paracelsus entdeckt Suizid als Krankheit! - vom Kranksein besessener Leute, vom Veitstanz und schreibt es seiner Astronomie zu, dass er Einsicht in die „unsichtbaren“, die psychischen Krankheiten gewinnt und dies Neuland abzustecken vermag (vgl. S I, 11: 132).

V Die überlieferten humoralpathologischen Krankheitsnamen sind seiner Überzeugung nach falsch orientierende Namen, weil die ihnen zugrundeliegende Theorie, Krankheit resultiere aus einer Unordnung im Säftehaushalt, falsch ist. Solche Namen sind aber nicht nur Bretter vorm Kopf, sondern auch Blender, Mimikry, Bluff. Der Gebrauch der wissenschaftlichen Terminologie ist vielfach nach dem Prinzip ,Sprache macht Leute“ ein Hochstaplerkauderwelsch. „es ist ein subtils ausreden, der nichts kan auf teutsch, disputir auf kriechisch, und der nichts kan auf latein, disputir auf allacuttisch, allmal mit frembden selsamen bossen her; das man nit verstet, das gibt gelert erfarn leut“ (S I, 7: 130).

VI Er sieht es aus Gründen der Klarheit und allgemeinen Verständigung für notwendig an, in den Synonymenwirrwarr, das Durcheinander und Flickwerk der Namensüberlieferung eine neue Ordnung zu bringen, Übersicht zu ermöglichen (vgl. S I, 11: 133). Kein Zweifel, dass er sich als den rechtmäßigen Namensgesetzgeber der gegenwärtigen Monarchei betrachtet. Dies wird dann im 18. Jahrhundert Linné werden.

VII Als der ,rechte grund', das angemessene namenschaffende Moment erscheint Paracelsus der Ort im Makrokosmos, der mit der Krankheit korrespondiert: der sie verursacht oder auch ihr Heilmittel ist. Wer eine Krankheit morbus hermodactyli nennt, also die ,Herbstzeitlosenkrankheit', morbus coloquintialis, das ,Kürbisgewächs', oder morbus elleborinus, die ,Christrosenkrankheit‘, hat etwas begriffen; er nennt die Stelle im Makrokosmos, mit der die Krankheit im Rapport steht. Der ist ein ,philosophus', d.h. ein Naturkundiger, der für die menschliche Krankheit Namen wählt, die den Durchblick auf die korrespondierende Stelle in der großen umgebenden Natur erlauben. Der Mensch ist eine Wiederholung des Makrokosmos, sein Kind und Erbe, eine ihn spiegelnde Karte und verkleinerte Schrift.

Ihr sollt nicht sagen: das ist cholerisch, das melancholisch - das wäre Säftelehre -, „sondern das ist arsenicus (Arsen), das ist aluminosisch (alaunartig)“. Wenn ihr sagt: das ist jovisch, das ist saturnisch, dann werde ich nicht mit euch streiten. Sagt ihr: das ist acorina 
aegritudo [wahrscheinlich von acorus calamus - eine schwertlilienähnliche Pflanze; „Kalmus-Krankheit"], und diese Krankheit ist anthera [wahrscheinlich von anthericum liliago, Graslilie], dann würde ich sagen: ihr seid gelehrte Doktoren, und sage die Wahrheit damit. Denn das geht aus der philosophei hervor. ${ }^{13}$

Eine richtige Nomenklatur der Krankheiten wäre ein Spiegel der Topographie des Universums. Paracelsus erläutert hier - darauf macht Wegener (1988: 108) aufmerksam - weniger seine Praxis als ein Erkenntnisideal (vgl. auch das Kapitel zur Signaturenlehre bei Klein 1992: 121ff.).

„da alle creata seind buchstaben und bücher, des Menschen herkommen zu beschreiben“ (S I, 12: 32). - Er formuliert eine Umschichtung in der Hierarchie der Bücher, untergräbt die Autorität der überlieferten Schrift, das Schriftprinzip, und fordert eine Orientierung an dem von Gott selbst geschriebenen Buch der Dinge. Die Umorientierung wird nicht zufällig durch die Buchmetaphorik ausgedrückt. In der Geschichte der ,Lesbarkeit der Welt‘ schreibt Paracelsus ein neues Kapitel, die res, die creata selbst werden Gegenstand fast schon einer Lesewut. Nicht das geschriebene Wort, das immer nur Schatten bleibt, nicht die Alten sind die Tür zur Arznei, sondern das Licht des von Gott diktierten Buches. Sein Verhalten diesem ,Licht der Natur' gegenüber ist anderer Art als die des messenden und rechnenden, experimentierenden Forschers, es ist die des Deuters einer Hieroglyphe. Er bleibt insofern, wie der Naturkundige des Mittelalters, ein Leser, Theophilologe, ein Liebhaber des Schöpfungswortes, sein Metier ist das der Hermeneutik. ${ }^{14}$

Aber das Spiel der Ähnlichkeiten, der Entsprechungen zwischen der kleinen Welt des Menschen und der großen des Kosmos, ist nicht mehr, wie es nach der faszinierenden Darstellung durch Foucault (1980), im zweiten Kapitel der Ordnung der Dinge, scheinen kann, ein statisches Spiegelverhältnis. ${ }^{15}$ Es gibt hier ein Moment der Unruhe; wenn ich richtig sehe, vielleicht sogar eine Unterwanderung des Prinzips der Analogie:

- Das erfahrungshungrige Auge, das nicht stillesteht.

13 Text nach der Übersetzung des Paragranum von Gunhild Pörksen, Paracelsus 1990: 33; vgl. S I, 8: 142.

$14 \mathrm{Zu}$ diesem Thema vgl. die hervorragende Dissertation Am Anfang war das Wort von Wolf Peter Klein 1992, das Kapitel B „Auslegungen des WORTs in der frühen Neuzeit“. Vgl. ebenfalls die schöne Arbeit von Wegener 1988.

15 Foucault, der sich in diesem Kapitel wiederholt auf Paracelsus beruft, scheint ihn nur via Sekundarliteratur zu kennen und trifft in seiner Charakteristik Hohenheims m. E. nur die halbe Wahrheit. 
- Ein Ausformulieren des Prinzips der Ähnlichkeiten und der zugehörigen Deutetechnik in die verschiedensten Richtungen, ein Bewusstmachen und Reflektieren darüber, das vielleicht schon an seinen Rand führt.

- Eine Verzeitlichung und Begründung des Kosmos. Statt der alten, z. B. bei Hildegard von Bingen vorherrschenden Denkfigur des Vergleichs, des ,wie dort - so hier‘, ein Einführen der das statische Spiegelverhältnis dynamisierenden Konjunktionen nachdem und auch weil, statt des additiven Stils der älteren Tradition die beginnende erklärende Hypotaxe.

- Eine Einführung von Begriffen, in denen er, mit langfristiger, oft indirekter Wirkung, die Entstehung der Welt und der Dinge vorstellbar zu machen sucht: Yliaster, Archaeus, die berühmten Tria Prima. Von den ,Drei Ersten“ wurde Sulphur unter dem Namen Phlogiston zu einem Schlüsselbegriff der Chemie des 18. Jahrhunderts, dessen Befragung wiederum zu einer Grundlegung der modernen Chemie beitrug (vgl. den Aufsatz „Die Produktivität eines Phantoms. Lichtenberg, das ,Phlogiston“ und die neue Chemie Lavoisiers“ in diesem Band). Und der Archäus gehört vermutlich in die Ahnenreihe der ,Lebenskraft‘.

- Schließlich, und damit zusammenhängend: Was uns aus der geschichtlichen Ferne als Analogie erscheint, als Vergleich oder spiegelbildliche Ähnlichkeit, war in den Augen des Paracelsus oft eher schon Identität. Die Welt ist vorgestellt als von einheitlich wirkenden Prinzipien durchwaltet: das alchemische Prinzip ist eines von ihnen, das magnetische ein anderes.

Das Bild einer durch das Gesetz der Ähnlichkeiten bestimmten, geschlossenen, sich in sich drehenden und spiegelnden Welt trifft also für Paracelsus nur zur Hälfte zu. Sein Erkenntnishunger treibt es bis zu einer Grenze vor, wo neue Fragen beginnen, sein Versuch, sich die Schöpfung prozessual in gleichsam naturwissenschaftlichen Begriffsbildern vorzustellen, ihr Werden wie Vergehen, unterhöhlt bereits den ganzen Bau der Ähnlichkeiten oder hat sich unbeabsichtigt so ausgewirkt.

Er bildet die Tradition um; der Stollen, den er in die Zukunft schlägt, ist dann freilich in einer anderen Richtung verlassen worden.

Ich kann dies Gebiet hier nur andeuten, es wäre ein eigenes Kapitel und soll in anderem Zusammenhang aufgegriffen werden. Die Stilfigur der praeteritio hat den Vorteil, dass man etwas ausdrücklich übergehen kann. Zurück zum Handwerklichen: 


\section{Der lateinisch-deutsche Fachwerkstil und die Lehnprägung durch das Lateinische}

Paracelsus in Basel ist ein berühmtes Kapitel, auch ein schönes Buch von RobertHenri Blaser. ${ }^{16}$ Während der elf Monate, die er sich dort hielt, von März 1527 bis zum Februar 1528, entfaltete er eine rege Vorlesungstätigkeit, zunächst auf lateinisch, dann auf deutsch bzw. in einer eigentümlichen Mischsprache. Ganz sicher können wir nur sein, wie er gesprochen hat, wenn von einer Vorlesung mehrere Hörernachschriften erhalten und diese stückweise identisch sind. Auf vier Vorlesungen möchte ich kurz eingehen.

Die Vorlesung De Tartaro - ein paracelsischer Begriff, der Steinleiden und überhaupt mineralische Schlackenbildungen im Körper meint - ist lateinisch überliefert. ${ }^{17}$ Aber dem Latein sind deutsche Rudimente eingesetzt, die in der Volkssprache anscheinend den Charakter geprägter Münzen haben:

et si aër non est tam fortis, ut possit pellere infectionem, so schlegts wider hinab und ligt im magen und drucket, unde est quod dicunt, es schüt mich nicht, sonder es druckt mich. (S I, 5: 55)

quare autem schüts heut und morgen nicht et suum periodum habet, quae causa? (S I, 5: 56)

Das Folgende scheint sprichwörtlich:

nam in dies habent digestionem, dan es ist kein baum oder ding, es hat im jar ein mal ein auswerfen, sic et faeces suas digestiones. (S I, 5: 56)

Hier folgt noch einmal volkssprachliche Symptomatologie:

Compressio stomachi) wan sie faul seind und verdrossen, lempen dohin, vix pedes tollere possunt aut os aperire, so faul und schwer: signum est in stomacho esse, nam in hepate et renibus sunt agiles. item quando dicunt, ich schmecke mich, eben als het ich herte eier gessen, signum stomachi. (S I, 5: 57)

16 Blaser 1979 hat den Casus nach den verschiedensten Richtungen durch subtile Detailforschung erhellt. Vgl. neuerdings auch die glänzende Biographie von Pirmin Meier 1993.

17 Neuerdings hat Wolfgang Schneider 1985 eine deutschsprachige Hörermitschrift der TartarusVorlesung ediert. Dass Paracelsus die Vorlesung lateinisch gehalten hat, wird Schneider (1985: 5) immer zweifelhafter: „Der neue Textbeleg bietet der Vermutung eine neue Stütze, dass Paracelsus die Tartarus-Vorlesung ebenso wie die chirurgische des vorangegangenen Semesters in deutscher Sprache gehalten hat. “ In dem Fall wären die in der lateinischen Mitschrift erhaltenen deutschen Einsprengsel Zitate aus der Volkssprache, sozusagen volkssprachliches Fachwerk. 
Das Deutsch ist in diesem Fall als Zitat eingefügt, als Redensart und Sprichwort, also zwei Formen mündlich gespeicherten Wissens. Während wir in der älteren Sachprosa, so volkstümlich sie auf uns wirken mag, keineswegs sicher sein können, dass in ihr mündliche Überlieferung mitwirkt, fassen wir hier geprägte Elemente der mündlichen Tradition; das quod dicunt deutet auf den Zitatcharakter. Es passt vielleicht zur Grundhaltung Hohenheims, der als Gelehrter auf mündlich geprägtes Laienwissen zurückgreift, dass er hier, einigermaßen singulär, wie mir scheint, gemünzte Patientensprache referiert, Ausdrücke, die er als Bestandteil der Symptomatologie und Diagnose ernst nimmt: ${ }^{18}$ eine möglicherweise aufschlussreiche Quelle, die an Barbara Dudens Untersuchungen von Selbstaussagen Eisenacher Patientinnen aus der Zeit um 1730 denken lässt. ${ }^{19}$

In einer Paragraphenvorlesung über Wurmkrankheiten beginnt er mit lateinischen Merksätzen, Paragraphus primus, Paragraphus secundus usf., die er vermutlich diktiert hat, dann folgen Erläuterungen, z.B.:

Paragraphus primus.

Vermium generatio tria principia habet, primum est ex nutrimentis, secundum ex mineralibus et tertium ex elementatis. (S I, 5: 213).

Secunda causa ex mineralibus; dan es möglich ist, das im fleisch, im blut, im mark würm mögen werden, die nemen ir principium aus keiner feulung, sonder wachsen ex mineralibus. es wird auch generatio vermium, so man sperma in einem wasser trinket oder isset oder von einem fischrogen, so ein sperma in exaltatione ist, generiren auch vermes. (S I, 5: 213)

In diesem Fall sind die lateinischen Ausdrücke meist Zitate, und zwar aus dem einleitenden Paragraphen 1, sie scheinen das Fachliche hervorzuheben. Zwei weitere Beispiele:

Wan einer auf ein fart würm empfindet und dan nicht mer, ist signum, das stomachus darauf geneigt sei zu generiren würm; und welchen der atem übel stinket, ist verissimum signum vermium und das die würm faulen, und die sind lang, dürr; [...]. wan nun würm ex mineralibus wachsen, so wachsen sie in stomacho. (S I, 5: 214)

18 Wir finden, worauf Michael Kuhn mich hinweist, bei Paracelsus allerdings auch deutliche Zweifel an der Patientenrede: „gibt dem arzet die zung des kranken etwas, so ists doch nichts volkomens, da wir mögen von uns selbs das wenigste semlin nicht erfinden“ (S I, 7:370).

19 Gemünzte Patientenrede als Dokument von Vorstellungen, die sich in der frühen Neuzeit Frauen und Männer von ihrem Körper und ihren Krankheiten bilden, wäre eben nicht nur für den Medizinhistoriker, sondern auch für den Sprachhistoriker eine erwünschte, seltene Quelle. Barbara Dudens Geschichte unter der Haut (1987), die zitatenreiche Krankengeschichten eines Eisenacher Arztes um 1730 zugrunde legt, bedeutet hier einen neuen Zugriff. - Sehr ergiebig wäre in dieser Hinsicht der Nachlass Samuel Hahnemanns. 
Alle würm wachsen ex spermate und nicht ex putrefactione, dan ein ietlich ding wachst ex semine. (S I, 5: 215)

Die Mischsprache hat hier anscheinend System, in der Weise, dass ein lateinisches Gelehrsamkeitsgerüst, als Fachwerk, mit deutschen Lehmziegeln gefüllt wird. Wie wurde dieser Text von Paracelsus gesprochen? Wir können keineswegs sicher sein, drei Möglichkeiten sind denkbar:

I. Er könnte ihn auf lateinisch vorgetragen haben und der Hörer ihn bis auf das, was er für das wichtigste Fachvokabular hielt, in sein Deutsch eingebettet haben. So schreiben wir z.B. mit, wenn wir einen englischen Vortrag hören: das Wichtigste auf englisch, den Rest deutsch.

II. Er könnte deutsch gesprochen und der Hörer das Fachliche auf lateinisch festgehalten haben, weil er nur in dieser Sprache an den fachlichen Inhalt gewöhnt und seiner sicher war.

III. Oder Paracelsus könnte in dieser Mischsprache gesprochen und selbst das Fachwerk eingezogen haben. Wie wenig sicher wir sein können, entnehmen wir z.B. der in zwei Mitschriften erhaltenen Vorlesung Über Wunden und Wundbehandlung. Der gleiche Passus findet sich hier in sehr verschiedenen Fassungen; es geht um die Verwundung der Hoden:

Hörer A: „Si testiculi tangantur, si non mitigetur, tunc venit magnus dolor in 24 stunden debent mitigantia apponi, si inflentur putrefiunt et venit ignis persicus.“

Hörer B: „Wenn aber die testiculi troffen werden, sol man von aller ersten mitigativa brauchen. dan es kompt ein solcher tumor, wenn man 24 stunden ubersicht und nit mitigirt, das es ad ignem persicum oder ad herniam get, und sind unseglich dolores." (S I, 5: 327, 329; Mitigativa sind Linderungsmittel)

Andrerseits gibt es eindeutige Indizien, dass Paracelsus auch selbst den zuerst zitierten Sprachtypus verwendet hat. Zwei Hörer einer Vorlesung über chirurgische Krankheiten, die stark voneinander abweichen, schreiben gemeinsam, es geht um Brustgeschwüre, folgenden Satz:

Ich sag das nacta sei apostema immaturum, das da kompt aus den mestrualischen flüssen, so die selbig durch salem plumosum, dem nach in ein coagulation gezogen, aus der selbigen in resolutionem. (S I, 4: 250f.).

Apostema immaturum (= ,unreifes Geschwür'), menstrualische flüsse, sal plumosum (=,gefiedertes Salz'), coagulation (=,Gerinnung'), resolutio (= ,Auflösung') sind offenbar Ausdrücke aus der Fachsphäre.

Ich möchte diesen Sprachtyp die ,Fachwerksprache“ nennen und meine damit, wie angedeutet, eine Sprachmischung, die dadurch charakterisiert ist, dass in die weniger feste Vernakularsprache oder Umgangssprache das feste 
Gerüst des bekannten Fachvokabulars eingezogen ist. Auf der Basis des ,Bilinguismus‘ des Vortragenden und seiner Hörer nimmt das Deutsche hier eine besondere geregelt gemischte Gestalt an.

Sie beschränkt sich im Fall Hohenheims nicht auf die Basler Vorlesungen, sondern charakterisiert, in zurückhaltender Form, sein gesamtes Schrifttum. Dies ist im Wesentlichen deutsch, aber die Titel seiner Werke, genauer, seiner Handschriften, sind überwiegend lateinisch und nicht immer einfach: Philosophia de generationibus et fructibus quatuor elementorum. Nicht selten sind lateinischdeutsche Mischtitel wie im Fall der Septem Defensiones: Verantwortung über etliche Verunglimpfung seiner Mißgönner oder der Practica deutsch, die kleinere Zahl hat einen nur deutschen Titel wie z.B. die Große Wundartzney. Auch die Kapitelüberschriften sind häufig lateinisch, hinzu kommt das Fachwerk seiner Terminologie und Nomenklatur im laufenden Text. Paracelsus war es durchaus bewusst, dass seine Sprache auf diese Weise ,gestrîfelt‘, ,gestreifelt‘ war, wie man im Spätmittelalter sagte. ${ }^{20}$

Es kommt vor, dass er sein Latein übersetzt. Der lateinisch-deutsche Doppeltitel ist dafür ein Beispiel, ein anderes das von ihm selbst, wie im einleitend besprochenen Fall der Schrift über das Bad Pfäfers, beigegebene Glossar. Nur teilweise gehören auch die bei Paracelsus wie seinen Zeitgenossen beliebten zwei- und mehrgliedrigen Wortgruppen hierher. Dieser Stilzug erfüllt verschiedene Zwecke. Häufig handelt es sich um das Verfahren der Synonymenvariation, das man als ein fortgesetztes Interpretieren, Verdeutlichen, Vereindringlichen beschreiben kann. Paracelsus setzt es gelegentlich ein, wo es darum geht, einen schwierigen spekulativen Sachverhalt oder neuartigen Begriff einkreisend zu umreißen, an anderer Stelle, um durch eine Art Wiederholung, im Sinn der amplificatio oder climax der Rhetorik, $\mathrm{zu}$ vereindringlichen. Dreigliedrig und

20 Thomasin von Zirklaria hebt in Der wälsche Gast (1216) das Lehrreiche dieses Verfahrens hervor:

33 Hie wil ich iuch wizzen lân, swie wol ich welhische kan, sô wil ich doch in min getiht welhischer worte mischen niht [...]

39 daz ensprich ich dâ von niht daz mir missevalle iht swer strîfelt sine tîusche wol mit der welhsche, sam er sol; wan dâ lernt ein tiusche man, der niht welhische kan, der spaehen worte harte vil, ob erz gerne tuon wil. 
zweigliedrig sind die gängigsten Syntagmen seiner Rede, „sein geist, sein liecht, sein englisch art“ (S I, 14: 116), „die natur der irdischen ding beschreiben und erkennen“ (S I, 8: 37). Beim letzteren Typ handelt es sich zuweilen um die reimende und stabreimende, mündlicher Tradition verpflichtete Paarformel, „zwilich und trilich“ (S I, 11: 152), „der nieren lieb und leid“ (S I, 8: 73); für uns besonders interessant ist das Nebeneinander von lateinischem Fachausdruck und seiner deutschen Übersetzung, die ,Zwillingsformel“ des Typs: „ein zeug und ein materia“ (S I, 12: 33), „mit kreften und virtutibus“ (S I, 14: 499), „nach irem bedünken, consilia, opiniones“ (S I, 11: 169), „mit was fug und bilicher concordanz“ (S I, 8: 84), „alle planeten haben im menschen ir gleich ansehung und signatur“ (S I, 8: 164; die letzten Beispiele nach Boehm-Bezing 1966: 62). Diese Formel, für die Peter von Polenz (1991: 215) in seiner Deutschen Sprachgeschichte weitere Beispiele nennt, ist für Paracelsus nicht besonders charakteristisch. Er war kein Gegner des lateinischen Fachwerks, im Gegenteil.

Lateinische Titel und Untertitel, Nomenklatur und Begriffsapparat, deutschlateinische Zwillingsformeln fügen das Werk ein in den Traditionsstrom gelehrter Schriftkultur. Titel wie Prognostication, Manuale, Defensio, Philosophia schließen es an an übliche Gattungsbezeichnungen, Disziplinenbezeichnungen, es gibt hier einen beachtlichen Reichtum, ohne dass eine klare Systematik erkennbar wäre. Sie bedürften, wie eingangs erwähnt, einer gründlichen Untersuchung. Das auch von ihm verwendete übliche Fachvokabular bildet die Brücke zur gelehrten Welt, so dass der Arzt, der Astronom, der Alchimist weiß, wo er sich jeweils befindet und wovon die Rede ist. Insofern knüpft Hohenheim an die Vorerwartung der Leser an; andererseits aber strapaziert er sie durch eigene neue Begrifflichkeit, die so befremdlich wirkte, das man schon im 16. Jahrhundert lateinische ParacelsusWörterbücher verfasst hat (vgl. Dorneus \& Bodenstein 1981; Toxites 1574).

Es handelt sich bei diesem Fachwerkstil um eine besondere Form der Zweisprachigkeit, um sprachinterne Diglossie. Unter ,sprachinterner Diglossie“ verstehe ich eine hierarchisch angelegte und funktional aufgeteilte Zweisprachigkeit innerhalb einer Sprache (vgl. zum Thema „Sprachen im Kontakt“ Weinreich 1977: 20ff.). ,Diglossie“ meint an sich das Nebeneinander zweier Sprachen in einer Gemeinschaft, wenn sie in verschiedenen Regionen oder von verschiedenen sozialen Gruppen bzw. Institutionen gesprochen werden. In Norddeutschland haben wir seit der Reformation die Diglossie von Niederdeutsch und hochdeutscher Schriftsprache, im Basel des 16. Jahrhunderts die von Latein und mundartlich gefärbtem Deutsch. Latein ist hier u.a. die Sprache der monastisch oder humanistisch geprägten Universität, die Sprache der gelehrten Sphäre, sein Gebrauch verbindet mit einer jahrhundertealten Tradition und einer universalen europäischen Gemeinschaft der Gelehrten, Deutsch ist dagegen die Sprache der Stadt und des Volks auf dem Land. Es sind zwei Kulturen, die Paracelsus problemlos ver- 
bindet, oft auch in einem schlichten Durcheinander von Latein und Deutsch zu vermischen scheint, und deren Gegensätze er andererseits wie kaum ein anderer zutage treten lässt. Was liegt hier vor? Wie lässt sich dieser Widerspruch erklären?

Als Paracelsus das Amt des Stadtarztes von Basel antritt, ausgestattet mit dem von der Stadt nicht ganz unumstritten verliehenen Recht, an der Universität Vorlesungen zu halten, gerät er in das Spannungsfeld zwischen ,town and gown“, wie man in Oxford sagt, und er tut alles, es in einen Konflikt zu überführen. Er las nicht in seiner ärztlichen Standestracht, sondern im Arbeitskleid, sah aus wie ein Fuhrmann, und auch sein Verkehr ging, heißt es, in diese Richtung (vgl. Bittel 1945; Blaser 1979: 37). In der schon erwähnten Intimatio vom 5. Juni 1527, dem als Flugblatt verbreiteten Vorlesungsprogramm, bricht er auf Latein mit der lateinischen Sphäre und arbeitet den Gegensatz zwischen den beiden Kulturen mit verwundender Schärfe heraus:

Auf der eigenen Seite die Berufung auf die Natur der Dinge und Erfahrung, eingeübten Gebrauch (man erinnere sich an die traditionsschwangere redensartliche Patientensprache!), eigene Arbeit und Erwägung, rei naturae und experientia, longus rerum usus, labor und ratio, insgesamt auf eine erfahrungsgebundene Realienkunde des Arztes, welcher sich auf Klassen, Ursachen und Symptome der Krankheiten und auf Heilmittel versteht.

Auf der Gegenseite das ängstliche Kleben an den Autoritäten Hippokrates, Galen etc., als handle es sich um Orakel. Er fasst seinen Angriff zusammen in dem Vorwurf der Rhetorik:

His enim autoribus [sic] splendidissimi quidem doctores, si diis placet, non autem medici nascuntur. Non titulus, non eloquentia, non linguarum peritia, nec multorum librorum lectio, etsi haec non parum exornent, in medico desideranda, sed summa rerum ac mysteriorum cognitio, quae una facile aliorum omnium vices agit. (Sudhoff 1936: 27)

Das in Basel angeschlagene Schmähgedicht, das als Antwort der Manen Galens aus der Unterwelt deklariert wird, greift den Hauptvorwurf auf (Sudhoff 1936: 38ff.):

Et tibi sum rhetor, sum modo mentis mops

Und dir bin ich ein Rhetor, ein Geisteschwacher.

Es kehrt den Spieß um, indem es Hohenheims Welt lächerlich macht, räumt ein, Galen kenne vielleicht nicht jene Bauernkräuter Zwiebel und Knoblauch, dafür aber die Nieswurz - ein Mittel gegen Geisteskrankheit. Von Theophrasts scharfer Stimme und wortarmer Zunge ist die Rede, der bäurischen Schar, rustica turba, die er in den Hörsaal ziehe. Man erwidert ihm mit seinen Waffen, parodistisch, populistisch: Er sei nicht würdig, dem Hippokrates das Nachtgeschirr nachzutragen oder Galen die Schweine zu hüten. 
Der Gegensatz zwischen den beiden Welten, einer durch jahrhundertelange Überlieferung erprobten Wahrheit und der durch das erfahrene Auge zu prüfenden Gewissheit, tritt durch Hohenheims Provokation und die Antwort der anonymen Manen Galens schärfstens an den Tag: Sie unterscheiden sich durch ihren Sprachbegriff und die Spielregeln der Wahrheitsfindung, also ihr Wissenschaftsparadigma, durch verschiedene Öffentlichkeiten, Trennung von Universität und Stadt oder Durchdringung der Stadt, für die Paracelsus deutsch liest, mit Wissenschaft. Es sind zwei soziale Felder und Lebensformen, zwei Sprachstile und am Ende auch zwei verschiedene Sprachen.

Beim Übergang der Wissenschaften vom Lateinischen in die Landessprache geht es nicht in erster Linie um die Sprache, sondern, wenn ich einen Ausdruck aus Jürgen Schiewes im Abschluss befindlicher Sprachgeschichte der Freiburger Universität vorwegnehmen darf, um den „Austausch zweier Denkstile“ (vgl. Schiewe 1996). Schiewe beschreibt den Sprachenwechsel der neuzeitlichen Universität als Funktionswandel. Er hat dabei tastende Andeutungen, die ich in dem Vortrag Ist die Sprache ein selbständiger Faktor der Wissenschaftsgeschichte? Über die Anstößigkeit unerwarteter Sprache gemacht habe (vgl. den Aufsatz in diesem Band), dadurch dass er sie mit der sozialgeschichtlich basierten Erkenntnistheorie Ludwik Flecks (1980) und empirischen universitätsgeschichtlichen Studien verbunden hat, $\mathrm{zu}$ einem weitreichenden, vorzüglich gesicherten Begriffsinstrumentarium ausgearbeitet, das mir hier wiederum als Anregung dient.

Der oben erwähnte Widerspruch scheint sich aufzuklären. Dass Paracelsus in Basel als Vorlesungssprache Deutsch wählte, war nicht nur Rücksichtnahme auf lateinunkundige Bader und Scherer, Erweiterung des Publikums, sondern eine Provokation im Sinn des Austausches der Denkstile: Er hoffte auf einen Umsturz und eine Neubegründung der Medizin. Paradox gesagt: Er hatte nichts gegen Latein, war sich aber bewusst, dass die alte Medizin in dem und durch das lateinische Sprachkleid institutionalisiert war.

Noch eindrucksvoller aber als dieser Fachwerkstil ist eine andere Konsequenz des Bilingualismus Hohenheims: der Abdruck des Lateinischen auf das Deutsche. Er verfügt selbstverständlich über beide Sprachen, wechselt von einer in die andere, wie man durch eine offene Tür von einem Zimmer ins andere geht. Wenn jemand auf diese Weise zwei Sprachen aktiv beherrscht und verwendet, spricht man von ,Bilingualismus‘. Paracelsus und seine studierten Zeitgenossen waren in diesem Sinn zweisprachig, bilingual, pendelten zwischen Latein und Deutsch hin und her. Dabei entsteht nun eine Mischsprache, die etwas anderes ist als sprachinterne Diglossie.

„Unser Herr Gott lest Filium suum predigen, sed excipitur sicut sol“, „Ich hab die Wort nicht gemacht, sed accepi“", sagt Luther in seinen Tischreden (Beispiele nach der grundlegenden Untersuchung von Birgit Stolt 1964: 220f.), Paracelsus: „Si non 
vult under dem pflaster hinweg gon, debet dari der trank“ (S I, 5: 361). - Statt einer hierarchisch angelegten, funktional aufgeteilten Zweisprachigkeit gab es hier eine ganz andere Form des Ineinander und Durcheinander, ein Missingsch und Kauderwelsch, wie es der Sprachkontakt bis in die Gegenwart hervorzubringen vermag und wie ihn die lateinisch-deutsche Sprachberührung häufig erzeugt hat.

In diesem Sprachklima entsteht nun aber auch leicht ein Übertrag lateinischer Muster auf die deutsche Sprache, besonders bei einem Autor wie Paracelsus, der in fortwährender Gedankenarbeit nach Ausdruck sucht und sich die Sprache auf bewegliche Weise gefügig macht. Vielleicht konnte er deshalb so rasch arbeiten, diktieren, und zwar oft in einer gedanklich konzentrierten und lebhaften, rhythmisch gegliederten und neuartigen Sprache, weil ihm die Sprache der Vulgata, als „Stilgrammatik“, in Fleisch und Blut übergegangen war und weil er in- und auswendig über einen enormen lateinisch oder auch deutsch geprägten Wissensvorrat verfügte, der ihm ad hoc zur Umformung zur Verfügung stand.

Die Prägung seines Deutsch durch die lateinische Unterlage reicht recht weit. Carolin Holtmeier (geb. Oberkrome) hat das in ihrer Staatsexamensarbeit Zur Sachprosa Theophrasts von Hohenheim vor dem Hintergrund des Lateinischen. Analyse einer chirurgischen Vorlesung in Basel (1992) auf sorgfältige Weise nachgewiesen. Sie wählt als Beispiel die Vorlesung Über Wunden und Wundheilung (S I, 5: 315-410), von der schon kurz die Rede war, und konzentriert sich auf die weniger eingedeutschte Nachschrift des Oporinus. Ihre erste Untersuchung gilt dem lateinischen Textgehalt (zwischen 40 und $70 \%$ ), dabei beobachtet sie in hohem Maße einen Bestandteil, den ich als lateinischen Fachwerkrahmen bezeichnen würde:

„1) Paracelsus gibt seiner Vorlesung einen lateinischen Rahmen durch lateinische Überschriften und ordnende Satzelemente.“ Gemeint sind die Überschrift Praelectiones chirurgiae de vulneribus, Unterüberschriften wie Cura, Nota, De signis capitis, satzgliedernde lateinische Adverbien und Konjunktionen.

„2) Er gebraucht darüber hinaus [...] Versatzstücke in großer Zahl, die er mit großer Wahrscheinlichkeit aus der früheren Medizinliteratur übernommen hat.“ Hier ist gedacht an Vokabeln der Dia gnose, signum est, non est letalis, an die durchgehend im Blick auf die Apotheker lateinisch formulierten Rezepte mit ihren Imperativen (unge, appone, debet dari), Maßangaben (unc., drach.), die Ausdrücke für alchemische Prozesse (destillatio, putrefactio), für Arzneien, ihre Zutaten und Wirkungsweisen (habet bonam virtutem, habet maximam virtutem), an formelhafte Verweise wie qui supra scriptus est und die Formulierung allgemeiner Regeln: unus charakter curat alterum.

„3) Er übernimmt sehr viele lateinische Worte als Fremdworte“, Fremdworte hier im Sinne des Fachausdrucks, z. B. für Organe und Körperteile, Krankheiten und Symptome, auch im Sinn des abstrakten Terminus. 
„4) Er bildet neue lateinische Abstrakta in adjektivischer und substantivischer Form.“

„5) Er zitiert frühere Autoren in lateinischer Sprache.“

Im Gegensatz zu Gisela von Boehm-Bezing (1966), die Paracelsus einer systematischen Darstellung nicht für fähig hält, erscheint ihr ,seine Vorlesung gerade durch das, was ich als Versatzstücke genannt habe, als wohlgeordneter und übersichtlicher Vortrag“ (Holtmeier 1992: 74).

Auf der anderen Seite untersucht Carolin Holtmeier am Text dieser Vorlesungsnachschrift den Abdruck des Lateinischen auf das Deutsche. Da gibt es die assimilierten Lehnwörter aus dem Lateinischen, Substantive wie circel, fistel, tenakel, balsam, defect, Adjektive wie chronisch, die Verben digeriren, mollificiren, geriren. Es gibt den Typus der Lehnübersetzung, welche das lateinische Kompositum Bestandteil für Bestandteil übersetzt, ein seit dem 9. Jahrhundert ungemein häufiger deutscher Wortbildungstyp (vgl. besonders Betz 1959 und Eggers 19631977, Bd. 1: passim). Der Durchblick auf die lateinische Unterlage ist in manchen Fällen überraschend: ,ingeweit‘ von intestina, ,zufälle‘ von accidentia, ,unempfintlich'von insensitivus, ,verharren'von permanere, ,zusamen ziehen'von contrahere, ,uberlegen'von superponere, ,einschütten'von infundere, ,ausstreken'von extendere, ,auftun“ von patefacere, ,ausdörren' von exsiccare. Wieviel von solchen Lehnwörtern und Lehnprägungen auf das Konto von Paracelsus geht, könnte nur nach eingehender Untersuchung festgestellt werden, erscheint mir aber, wie in der Einleitung ausgeführt, auch als unwichtig. Interessant ist die prägende Wirkung des Lateinischen, die hier beobachtet wird, auch in den verschiedenen Typen von Komposita, die zweigliedrig (fleischwunden, salzwasser), dreigliedrig (lindenblutwasser, weidwundentrank) als Mischkompositum (vulnus-salben) vorkommen, die häufige synonymische Zwillingsformel (balsam id est stichpflas ter; putrefactio id est, feult stinkt; coopertorio, id est hafendekel). Schließlich gibt es einige Anlehnungen an grammatische Konstruktionen und die Syntax des Lateinischen, die Übernahme des A.c.I. oder lateinischer Partizipialkonstruktionen, des Infinitivs, wofür sich bei Boehm-Bezing (1966: 28-33) reiches Belegmaterial findet.

Wie ein neuer Schlüssel zur Sprache Hohenheims erschien es mir, als Carolin Holtmeier in einem Paracelsus-Seminar (des Sommers 1991) die Dritte Defension in lateinischer Übersetzung vorlegte. Sie wirkt wie eine Rückübersetzung und entstand, nachdem sich ihr bei ihrer Untersuchung der Eindruck einer lateinischen Unterlage des paracelsischen Deutsch eingestellt hatte. Ich lege hier einen größeren Passus vor, auch wenn sein interlinear wiedergegebenes Latein klassischer sein dürfte als das von Paracelsus gebrauchte: 
Die drite defension

Tertia defensio

von wegen der beschreibung der neuen recepten

de descriptione receptorum novorum

Aber uber das und gemelt ist, ist das geschrei noch größer

De quo autem quod nuntiatum est clamor maior

erstanden under den unverstendigen vermeinten und erdichten

ortus est inter imprudentes et opinatos et fictos

arzten, die da sagen das meine recept so ich schreib ein gift,

medicos, qui dicunt recepta mea, cum scribo: venenum,

corrosiv und extraction sein aller bösheit und giftigkeit der

corrosivum et extractio, esse totius malitiae et acerbitatis

natur. auf solch fürgeben und ausschreien were meine erste

naturae. talibus proditis et exclamationibus esset prima

frag, so sie zu antworten tüchtig weren ob sie wißten,

quaestio mea, si respondere periti essent, an scirent,

was gift oder nit gift sei? oder aber ob im gift

quid venenum aut non venenum esset? Aut, an in veneno

kein mysterium der natur sei?

nullum mysterium naturae esset?

dan im selbigen puncten sind sie unverstendig und unwissend

Nam in eodem puncto ii sunt imprudentes et inscii

in den natürlichen kreften. dan was ist, das got erschaffen

virium naturalium. Nam quid est, quod deus creavit,

hat, das nit mit einer großen gaben begnadet sei dem menschen

quod non magno donodonatumsit homini

zu guten? warumb sol dan gift verworfen und veracht werden,

bonum? Cur venenum reicendum et contemnendum est, so doch nicht das gift sonder die natur gesucht werde?

cumnon venenumsed natura quaeratur?

ich wil euch ein exempel geben, mein fürnemen zu verstehen.

Vobis exemplum dem ad propositum meum intellegendum.

sehent an die krotten, wie so gar ein vergift und unlustig

Adspicite bufonem, quam acerbus et invitus

tier es ist, sehent auch darbei an das groß mysterium,

sit, adspicite simul illud magnum mysterium,

das in ir ist betreffend die pestilenz. solt nun das

quod in eo est ad pestilentiam. Num illud

mysterium veracht werden von wegen der giftikeit und

mysterium contemnendum est propter acerbitatem et

unlustikeit der krotten, wie ein großer spot wer das?

inertiamque bufonis? Quantus ludus esset?

wer ist der da componirt hat das recept der natur?

Quis est, qui composuit receptum naturae?

hat es nicht got getan? warumb wolt ich im sein compositum

Nonne deusfecit? Cur eius compositum 
verachten, ob er gleich zusamen sezet das mich nicht contemnam, cum composuerit, quae mihi non genug dunket? ist es der, in des hant alle weisheit satis videntur? is est, cuius in manu tota sapientia stehet, und weißt, wa er ein ieglisches mysterium sistat, qui sciat, quo omne mysterium hinlegen sol. warumb wil ichs mich dan verwundern oder deponendum sit.Cur id mirer vel scheuen lassen? darumb das ein teil gift ist, recusem? Propterea quod unum venenumest den andern mit dem verachten? ein ietliches ding alterum una cum eo contemnendum est? omnibus sic sol gebraucht werden, dahin es verordnet ist, utendumest, ut praecepta sint, und wir sollen weiter kein scheuen an demselben tragen, neque porro nobis vericundia eiusdem opus est, dan got ist der recht arzt und die erznei selbst. quod deus est rectus medicus et medicina ipsa. es sol ime auch ein ietlicher arzt eingebildet lassen sein Omnis medicus noverit die kraft gottes die Christus uns zu verstehen gibt vim dei, quam Christus nobis significat sprechende: und ob ir werden gift trinken, es wird euch dicens: et si venenumbiberitis, (vobis) nit schaden. so nun das gift nicht uberwint sonder non nocebit.si venenum non superat, sed gehet on schaden, in dem so wir es brauchen nach verordneter sine detrimento exit, cum eo utimur praecepto art der natur, warumb soft dan gift veracht sein? modo naturae, cur venenum condemnendum est? der gift verachtet, $\quad$ der weiß umb das nit das im gift ist. qui venenum contemnit, id nescit quod in veneno est. dan das arcanum, so im gift, ist gesegnet dermaßen, nam arcanum, quod in veneno (est), fortunatum est eo modo, dasim dasgiftnichts nimpt nochschat nicht ut neque ab eo venenum quicquam auferat neque noceat. neque aber das ich wölle euch mit disen versal und paragrapho vero volui vobis his versibus et paragrapho zufrieden gestelt haben oder genugsam mich defendirt, satisfecisse vel satis me defendisse, sonder weiter ein merern bericht euch fürzuhalten notwendig sed etiam altera explanatio (vobis) opus ist, so ich doch das gift genugsam erkleren sol. est, quia venenum satis mihi exponendum est.

Der Eindruck war: Wenn sich das so gut, so Wort für Wort auf lateinisch sagen lässt, in manchen Wortbildungen und Satzkonstruktionen analog, ist dann auch 
das Deutsch des Paracelsus in viel höherem Grad eine Lehnbildung als wir wissen? Einmal darauf aufmerksam, glaubt man es an immer mehr Stellen durchzuhören, durchschimmern zu sehen, als sei das Deutsche die Rückseite der lateinischen Tapete.

Latein und Deutsch sind nicht nur zwei Sprachen, sondern im Jahr 1527 auch zwei sozial verankerte Denkstile. Die beiden Formen der Zweisprachigkeit, von denen hier die Rede war, ,sprachinterne Diglossie ‘ und Mischsprache, gehen bei Paracelsus ineinander über und durcheinander, sie werden hier von mir bewusst idealisierend auseinandergehalten. Es sind unterschiedliche Typen.

Dass alle Gelehrsamkeit, überspitzt gesagt, zunächst lateinisch war, bedeutet, dass das in den deutschen Sprachkörper eingezogene Latein innerhalb eines Textes Zweisprachigkeit aufrecht erhielt und durch das Rahmenwerk an die Sphäre der Gelehrsamkeit, ihre Autorität, ihren Talar erinnerte, dass sich an ein lateinisches und griechisches Wort - und das gilt bis ins 20. Jahrhundert - die Vorerwartung richtete, es mit einem wissenschaftlichen Fachausdruck zu tun zu haben. Die Gelehrtensphäre bleibt der deutschen Sachprosa eingesenkt.

Der lateinische Hintergrund der Gelehrsamkeit hat aber auch zur Folge, dass die deutsche Sachprosa sich im Hin und Her zwischen diesen Sprachen, als Lehnbildung der lateinischen Schriftkultur herausbildet. Fachwerkstil und Lehnprägung unserer Sprache reichen weit in die Neuzeit, die sich so gern im Bild der Emanzipation begreift. Dass auch Paracelsus dafür ein herausragendes Beispiel sein könnte, eines, dem diese Imprägnierung zugutekommt, war mir neu und ist weiteres Hinschauen wert.

\section{Sermo humilis}

In einer Vortragsreihe, „Paracelsus (1493-1541). Versuch einer Annäherung“, die das Freiburger Studium Generale im Sommersemester 1993 veranstaltete, wurden zur Einführung von Manfred Schradi die Septem Defensiones vorgetragen. Es war ein denkwürdiger Abend. Das lag gewiss zuerst an der Kunst des Interpreten, aber auch ein wenig an der mimischen Partitur des Textes. Die Situation des mündlichen Vortrags ist ihm eingeschrieben. Die Zuhörer folgten einer 500 Jahre alten Sprache, als sei sie ihnen vollkommen durchsichtig und entstamme ihrer Gegenwart.

Paracelsus sei leicht zu sprechen, meinte Dr. Schradi anschließend, ein mündlicher Autor. Die Rhetorik mache es leicht; vermutlich habe er diktiert.

In der Tat ist der rhetorische und gesprochene Bau der Defensiones in jedem Satz spürbar. Das Gegenüber von ,Ich' und ,Ihr‘ ist die Grundfigur des Textes: Auf der einen Seite der Autor, der die sieben Pfeile der Verunglimpfung in sein Bild 
übernimmt, auf der anderen die Schar seiner Widersacher. Verantwortung über etzlich Verunglimpfung seiner Mißgönner, Verteidigung vor einer Hörerschaft ist die Situation der Rede.

In der oben deutsch und lateinisch wiedergegebenen dritten Defension lassen sich fast alle rhetorischen Mittel finden, die man auch sonst bei Paracelsus antrifft. Fast in jedem Satz findet sich jener mehrgliedrige Ausdruck, die Zwillings- oder die Drillingsformel, dem Gisela von Boehm- Bezing (1966) den größten Teil ihrer Stiluntersuchung gewidmet hat. Eine rhythmische Wiederholung kommt durch die gleiche oder annähernd gleiche Silbenzahl der Glieder in den Satzbau; das Isokolon, „die Koppelung gleich langer Satzglieder“ (Curtius 1984: 84; vgl. Norden 1958: 813ff.), ist eines der ältesten Mittel der Poesie und rhetorisch bestimmter Prosa. Boehm-Bezing (1966: 70) macht darauf aufmerksam, dass dieses schlichte Mittel nicht zum ornatus gehört:

ist das geschrei noch größer erstanden, under den unverstendigen vermeinten und erdichten arzten, die da sagen das meine recept so ich schreib ein gift, corrosiv und extraction sein aller bösheit und giftigkeit der natur. auf solch fürgeben und ausschreien were meine erste frag, [...].

Es folgt eine Fragenreihe, wie wir sie etwa aus den Paulinischen Briefen (vgl. z. B. 1. Korinther 9) oder aus der Bergpredigt (Mt 6) kennen. Der nahezu gleichförmige Bau der Sätze, eingeleitet durch ein wiederkehrendes Fragewort, diese Wiederholung der mit dem ähnlichen oder identischen Satzbauplan gegebenen rhythmischen Hohlform der Sätze, erzeugt jenen Parallelismus, den wir aus der Bibelsprache kennen und der Paracelsus besonders naheliegt: „ob sie wißten, was gift oder nit gift sei? oder aber ob im gift kein mysterium der natur sei?“ „warumb sol dan gift verworfen und veracht werden [...]?“ „warumb wolt ich im sein compositum verachten, [...]?“ „warumb wil ichs mich dan verwundern oder scheuen lassen?“ Das einleitende Fragewort tritt an die Stelle der auch sonst von Paracelsus geliebten Anapher, des wiederkehrenden Einleitungswortes gleichgebauter Sätze.

In dieser Weise verwendet er z.B. die Höreranrede, die Apostrophe, das predigthafte ,seht': ,ich wil euch ein exempel geben, mein fürnemen zu verstehen. sehent an die krotten, wie so gar ein vergift und unlustig tier es ist, sehent auch dabei an das groß mysterium, das in ir ist betreffend die pestilenz“. Die Stelle ist eine Kontrafaktur zu den exempla der Bergpredigt „Sehet die Vogel vnter dem Himel an“ - „Respicite volatilia caeli“, „Schawet die Lilien auff dem felde“ „Considerate lilia agri“ (Mt 6, 26 u. 28; Luther nach der Ausgabe Wittenberg 1544), deren Anführung Fragen folgen wie in dem paracelsischen Text.

Dem Anklang an die neutestamentliche Predigt folgt das direkte Zitat: „es sol ime auch ein ietlicher arzt eingebildet sein lassen die kraft gottes die Christus uns 
$\mathrm{zu}$ verstehen gibt sprechende: und ob ir werden gift trinken, es wird euch nit schaden“. Der Vers heißt in Luthers Übersetzung: „Vnd so sie etwas tödlichs trincken / wirds jnen nicht schaden. auff die Kranken werden sie die Hende legen / so wirds besser mit jnen werden“ (Luther nach der Ausgabe 1544), in der Vulgata: „et si mortiferum quid biberint, non eis nocebit: super aegros manus imponent, et bene habebunt“ (Mk 16, 18).

Bei einer so sichtbaren lateinischen, biblischen Unterlage, über die Paracelsus ,by heart‘ verfügt haben dürfte, ist es kein Wunder, wenn der deutsche Text, wie im vorigen Abschnitt ausgeführt, leicht zur Rückseite der lateinischen Tapete wird. - Der Dualismus im ganzen Stück, hier Ich - dort die Widersacher, kehrt wieder in der Antithese, die dem Parallelismus der Sätze als Gegensatz zwischen Gift und Mysterium der Natur, Anerkennung und Verachtung eingeschrieben ist. Die Verbindung von Parallelismus und Antithese ist traditionsreich, es liegt nahe, die Wucht des Parallelbaus von Sätzen oder Satzabschnitten durch die der Antithesen zu verstärken.

Hier freilich ist es die coincidentia oppositorum, das Paradox, das Paracelsus im Auge hat. Das Gift ist creatum und Heilmittel. Als Beispiel wählt er, gewiss nicht zufällig, sondern als bewusste Kontrafaktur selbst noch zum neutestamentlichen Vorbild, das niedrigste Tier, die Kröte, die nicht lustig anzusehen ist wie der Baum des Paradieses, sondern „,vergift und unlustig“: „,sehent auch darbei an das groß mysterium, das in ir ist betreffend die pestilenz“. Dass das verachtete, als giftig, gefährlich und neidisch geltende Tier zugleich Zauberkräfte auszuüben vermag und in der Volksmedizin als giftziehendes Mittel angesehen wird, ist vielfach belegt: „Wenn eine Kröte gespisset und ausgetrucknet wird, ziehet sie zur Pestzeit das Gift aus dem Menschen, so oft sie auf die Pestbeule geleget wird“ (Handwörterbuch des deutschen Aberglaubens, Bd.5, Sp. 618). Das Beispiel der Kröte gibt nicht nur einen Hinweis auf die paradoxale Natur von jedem venenum, was bekanntlich ,Gift‘ oder ,Heilmittel' bedeutet, sondern auch auf das Stilniveau, die Natur der Sprache, der Paracelsus den Vorzug gibt. In der niedrigsten Gestalt hat der, „der da componirt hat das recept der natur“, ein Mysterium verborgen.

In den fast durchgängig mehrgliedrigen Ausdrücken und der Fragereihe, dem Parallelismus der Sätze und der den Text bestimmenden Antithese, die zur wiederkehrenden Wortwahl führt, steckt rhythmisch und klanglich ein eindringliches Moment der Wiederholung in unserm Text, auch eines der Redundanz. Die Eigentümlichkeit Hohenheims, positiv Ausgedrücktes noch einmal negativ zu sagen, die „Wiederholung durch Negation und Position“ (von Boehm-Bezing 1966: 78ff.), findet sich auch mehrfach hier: „ein ietliches ding sol gebraucht werden, dahin es verordnet ist, und wir sollen weiter kein scheuen an demselben tragen“; „so nun das gift nicht uberwint sonder gehet on schaden, in dem so wir es brauchen nach verordneter art der 
natur, warumb solt dan gift veracht sein?“ Wenn man freilich genau hinsieht, sagen die inhaltsgleichen Glieder nicht dasselbe. Man beobachtet nirgends in unserem Text eine starre Wiederholung, weder auf der syntaktischen noch auf der verbalen Ebene, keine Stereotypie, sondern, was nicht mehr i.e. vorgeführt werden soll, ein leichtes, gar nicht kunstloses Spiel der Variation. Es ist die einfache Gebrauchsrhetorik der Predigt, nicht die der scholastischen oder humanistischen Schule, die Paracelsus hier nach den Regeln ihrer Kunst einsetzt.

Seine Sprache ist aus Elementen verschiedener Herkunft zusammengesetzt; eines, das noch kurz zu besprechen ist, ist das der Mündlichkeit. In den Seminaren über Paracelsus war es ein fast sicheres Mittel, wenn wir an einer schwierigen Textstelle festsaßen, sie laut zu lesen, am sichersten, wenn jemand in der Lage war, sie auf Schweizerdeutsch vorzutragen. Erst mündliche Intonation und mundartliche Einfärbung machten Gedankengliederung und Satzbau durchsichtig.

Ein krasses Beispiel solcher Mündlichkeit ist die bekannte Schlussrede des ersten Buches der Grossen Wundartzney von 1536, in der Paracelsus sein gemeines Landdeutsch entschuldigt. Sie war vielleicht schon dem ersten Schreiber und Setzer nicht ganz deutlich und lautet in der Erstausgabe:

\begin{abstract}
Ich hab ye unnd ye von hertzen begert / fürzůkommen der krancken verderbung / hoff mir werd es niemand verargen / ob geleich wol vil widder mich geredt wirt / so geschichts doch on erfarnuß / was ist darinn / nicht Phariseer / gleißner und schreyber sind / die allezeyt spaltung und zerreyssung zů machen genaygt sind / Ich habe inn disem Bůch den Artzeten ein Genůgen gethon / umb die anderen ist mir kayn sorg / ich hab hierin bißher ein låndtlichen spruch gefưrt / das mich kayner Rhetorick noch subtiliteten Berümen kan / sonder nach der zungen meiner geburt / und landssprachen / der ich bin von Ainsidlen / des lands ein Schweytzer / soll mir mein lendtlich sprach niemandts verargen / ich schreyb nicht von der sprach wegen / sonder von wegen der kunst meiner erfarenhayt / [...] (Paracelsus 1989, I: LXII).
\end{abstract}

Der Eindruck einer holprigen Mündlichkeit entsteht vor allem durch den Satzbau. Es gibt keine logisch gegliederten, durchkonstruierten Satzbögen, sondern ein Nebeneinander von Hauptsätzen, die von wenigen Nebensätzen unterbrochen sind. Er scheint anfangs mehrmals aus der Konstruktion $\mathrm{zu}$ fallen; vielleicht ist es aber die Konstruktion apo koinou, wo von einem Mittelglied Vordersatz und Nachsatz abhängig sind, die er verwendet? „hoff mir werd es niemand verargen / ob gleich wol vil widder mich geredt wird, so geschichts doch on erfarnuß“: Wenn auch viel gegen mich geredet wird, hoffe ich, es werde mir niemand verargen, geschieht es ohne eigene Erfahrung. Sudhoff interpunktiert und schreibt: „ich habe ie und ie von herzen begert, fürzukomen der kranken verderbung, hoff, mir werd es niemant verargen. ob geleich wol vil wider mich geret wird, so geschichts doch on erfarnus“ (S I, 10: 199). Das schafft Klarheit, ist aber nicht zwingend, differenzierter ist die obige Lösung. 
Das gilt auch für das Folgende, in Sudhoffs Transkription: „was ist darin? nichts. Phariseer, gleisner und schreiber sind, die allezeit spaltung und zerreißung zu machen geneigt sind“. Schöner wäre es, mit der Erstausgabe zu lesen und zu interpunktieren: „,was ist darinn? / nicht Phariseer / gleißner und schreiber sind? / die allezeyt spaltung und zerreyssung zůmachen genaygt sind?“

Die in der Regel zitierte, fast verbindliche Sudhoffsche Ausgabe ist bekanntlich keineswegs zuverlässig, ihre Interpunktion schafft ein Zuviel an logischer Klarheit. Diese fehlt in vielen Fällen der mündlichen Partitur des Textes, der erst durch richtige Betonung seine klare und eher schwebende Gliederung erhält. Er hat überdies Auslassungen, die für unsere Lesegewohnheit Unklarheiten des Bezuges schaffen können; wir sind Verdeutlichungen gewohnt, wie sie im Folgenden in eckige Klammern gesetzt sind:

Ich habe inn disem Bůch den Artzeten ein Genůgen gethon / umb die anderen ist mir kayn sorg / ich hab hierin biß her ein låndtlichen spruch gefưrt / das mich kayner Rhetorick noch subtiliteten Berümen kan / sonder [ich rede, ich verhalte mich] nach der zungen meiner geburt / und landssprachen / der ich bin von Ainsidlen / des lands ein Schweytzer / [darum] soll mir mein lendtlich sprach niemandts verargen / [denn] ich schreyb nicht von der sprach wegen / sondern von wegen der kunst meyner erfarenhayt /.

Kein Zweifel, scheint mir, dass der ,offene‘ Text der reizvollere ist.

Eine schlichte Antithetik, wie wir sie auch an diesem Text beobachten können, eingeübte Paarformeln und wiederkehrende Redensarten, parataktische Reihung an Stelle der Hypotaxe, scheinbare oder tatsächliche Fügungsbrüche, Anakoluthe, Ellipsen, „Mangel“ an logischer Struktur, Bezugsunklarheiten und die Wiederholung des Formelhaften - all dies gilt als Kennzeichen mündlichen Stils. Dass oft erst die Versetzung in den Duktus mündlicher Rede einen Text Hohenheims verdeutlicht, mag durch zwei weitere Beispiele belegt werden. Das erste entstammt dem Herbarius, die Rede ist von deutschen Ärzten:

wan es nur den namen hat, das ist das, so sind sie zufriden; es gang sonst wie es wol, so haben sie alle mal wol gehandelt und nichts verderbt und beston wol nach irem gedunken, so es alles nichts ist, dan ein falsch und ein ubersehen aus großem unverstant (S I, 12: 5).

Das zweite so wird durch den Kontext in der Weise umgeprägt, dass wir es durch die Konjunktion obwohl ersetzen würden.

Das zweite Beispiel, dem Paragranum entnommen, zeigt den Übergang von schlichter Rhetorik zu mündlicher Alltagsrede:

dan ein alchimist ist der becke in dem so er brot bacht, der rebman in dem so er den wein macht, der weber in dem das er tuch macht. also was aus der natur wachst dem menschen zu nuz, derselbige der es dahin bringt, dahin es verordnet wird von der natur, der ist ein 
alchimist. auf solches nun so wisset ein solche underscheid mit diser kunst, das zu gleicher weis als, so einer neme ein schafshaut und legt sie so rohe an für einen belz oder für einen rok, wie grob und ungeschickt das ist gegen dem kürsner und tuchmacher, also grob und ungeschickt ist es, so einer aus der natur etwas hat und daselbig nicht bereit, und mer grob und ungeschikter; dan es trifft ane gesuntheit den leib und das leben. (S I, 8: 181)

Die Elemente einer manchmal holprigen Alltagsrede, teils durch das Diktieren verursacht, und einer einfachen „natürlichen“ Rhetorik mischen sich an vielen Stellen. Hinzu kommt das Ingrediens einer gelegentlich unwegsamen Gelehrsamkeit. Paracelsus hat sich weder den Spielregeln der neuen Schriftlichkeit, des Buchdrucks, gefügt noch war es sein Ehrgeiz, „den Clavam und Herculis kolben inn henden“ $z u$ halten, „Namlich einen zirlichen stilum zuschreiben“, wie einer seiner Nachfahren sich ausdrückt. Es ist überraschend, dass Gisela von BoehmBezing und Joachim Telle sich so sehr schwertun mit der Einordnung einer Schreibart, die so leicht als sermo humilis zu identifizieren ist und als eines ihrer starken Beispiele in deutscher Sprache gelten kann.

Alles, was von Boehm-Bezing ausführt, um Stil und Syntax Hohenheims zu charakterisieren, ist Merkmal jener seit der beginnenden Geschichte des Christentums in der Spätantike traditionsreichen niederen Schreibart: die Ablehnung der Rhetorik und ihrer Feinheiten, ihres Schmuckes und Gepränges, und, dagegen gesetzt, die Berufung auf Inspiration und den einfältigen Geist des Evangeliums (von Boehm-Bezing 1966: 6-11), der geradlinige parataktische Stil und die eher bescheidenen Ansätze zur Hypotaxe, die Neigung zu Zwillingsformeln, Drillingsformeln, ohne dass diese Teil des ornatus wären (von Boehm-Bezing 1966: 37-75), der Hang zum Asyndeton (von Boehm-Bezing 1966: 57f.), zur schlichten, manchmal exzessiven Aufzählung (von Boehm-Bezing 1966: 73ff.), zu Parallelismus und Anapher (von Boehm-Bezing 1966: 82ff.) und zur Antithese. Unverständlich, dass sie das Resultat aus ihrer so sorgfältigen Bestandsaufnahme der Stilmittel herschenkt, indem sie diese Mittel nirgends auf einen Textzusammenhang, eine Autorenintention bezieht, sie nicht aus einer Stiltradition begreift und von allen Inhalten absieht.

Erich Auerbach (1958: 25-53) hat in seinem Aufsatz Sermo Humilis den schönsten Kommentar zu Hohenheims Stil geschrieben; er gibt darin eine Beschreibung christlicher Rhetorik, christlicher Umprägung der antiken Lehre von den drei Stilarten, die sich wie eine unmittelbare Charakteristik der paracelsischen Schreibart liest. Dabei ist weniger von den Stilmitteln die Rede als von einer neuen Haltung zur Welt, die in dem von dem gebildeten Heidentum der antiken Welt verachteten neutestamentlichen sermo einen Ausdruck gefunden hatte.

„Es ist eine praktische Gebrauchsrhetorik der Predigt, gemischt aus Feierlich und Alltäglich, zum Zwecke des Lehrens und Mahnens“, schreibt Auerbach (1958: 28) über eine Augustinische Predigt, und über Hieronymus: „Es ist eine Mischung 
von Erhabenheit und volkstümlicher Rhetorik und sich neigender caritas, eindringlich lehrend und szenisch belebt, für eine beliebige und unausgewählte Hörerschaft bestimmt“ (Auerbach 1958: 43).

Die Lehre von dem erniedrigten Gott, die sich an die Unmündigen und Armen im Geiste wandte, kehrte die antike Lehre, dass die Abstufungen des hohen, mittleren und niederen Stils sich an einer Hierarchie der Gegenstände auszurichten hätten, in der Weise um, dass sie diese Abstufungen nur noch an den Absichten des Redners orientierte, der sich als Lehrender des sermo humilis, als Mahnender des gemischten, mittleren Stils und als leidenschaftlich Aufrufender des sermo gravis bediente, und indem sie es zum Kern ihrer Auffassung des Wirklichen machte, dass sich in der Niedrigkeit das Erhabenste und Tiefste verbergen kann (vgl. Auerbach 1958: 30-40).

\begin{abstract}
Aber der niedere Stil der Heiligen Schrift schließt das Erhabene ein. Er enthält einfache, zuweilen alltägliche und kraß realistische Worte, er enthält unelegante und umgangssprachliche Satzbildungen; aber der Gegenstand ist erhaben, und die Erhabenheit zeigt sich in seiner Tiefe; überall enthält die Schrift verborgenen Sinn. In dieser Verschmelzung des Erhabenen mit dem Niedrigen wird das Erhabene (res excelsa et velata mysteriis, secreti sui dignitas) sehr häufig mit dem Dunklen und Verborgenen gleichgesetzt. Aber auch dies ist nicht in der Weise erhaben, daß nicht jedermann daran teilhaben könnte. Das Allumfassende dieses Stils ist die Demut (Auerbach 1958: 43).
\end{abstract}

Der christliche Geist habe eine solche lebendige Rhetorik erst wieder möglich gemacht, nachdem die alte im Formalismus erstarrt gewesen sei (Auerbach 1958: 44). ${ }^{21}$ Sie umfasste nicht nur Realistisches, sondern auch Vulgäres und Satirisches, menschlich Nahes, und modelte das Niedrige um im Zusammenhang des Ernsten und Hohen. „Das Polare, die Weite des Pendelausschlags sind wesentlich und entscheidend“ (Auerbach 1958: 46).

Auerbach spricht von der Heterogenität der Sprachelemente, den künstlichen lateinischen Neubildungen, vom Typ ,sanctificantur‘. „Sie alle haben etwas Ungeschicktes, Halbgebildetes, holprig Pedantisches; und zugleich, durch den Geist, der von ihnen ausgeht, etwas überwältigend Einheitliches“ (Auerbach 1958: 48). „[...] damals war es gleichgültig, ob der einzelne Teil des Sprachstoffes aus der Alltagssprache oder aus der halbgelehrten Bemühung um Ausdruck für die neuen Inhalte, oder am Ende aus griechischem oder semitischem Einfluß stammte; er wird eingeschmolzen in die Sprache der christlichen Gemeinschaft“ (Auerbach 1958: 49).

21 Vgl. neben Auerbach 1958 zu diesem Thema auch den Aufsatz über das Eingangsgebet zum Willehalm Wolframs von Eschenbach von Friedrich Ohly 1961/62. 
Der Autor Paracelsus scheint mir in dieser Tradition zu stehen. Die Mittelpunktstellung des Ich und die abrupte Verwerfung der Tradition, von denen in den Abschnitten 1 und 2 die Rede war, stehen historisch in Spannung dazu, waren aber in seinen Augen Teil seiner apostolischen Sendung und damit des Sermo humilis. Alles bisher von ihm Angeführte, von der ,stamleten zunge' bis zur Gebrauchsrhetorik, Gelehrtheit und scheinbaren Mündlichkeit, weist in diese Richtung. Auch hier liegt etwas überwältigend Einheitliches vor. Seine Stärke liegt in seinen Inhalten und seiner sachlichen Leidenschaft, in der Energie, mit der er die Tradition aufgreift und umdenkt, und in der lebendigen Spannweite, die sein Ich dabei gewinnt. Seine Sprache hat eine eigentümliche Ausstrahlung; wenn man sie laut liest, entwickelt sie eine starke Nachwirkung: ein Radium, das noch immer nicht zu Blei zerfallen ist. Der Grund dieser Sprache ist der göttliche Grund der Welt. „dan nichts ist beschaffen, das nit dem menschen zu ergründen sei, und darumb beschaffen, das der mensch nit müßig gang, sonder wandel in gottes, das ist in seinen werken“ (S I, 14: 116). - Darin präludiert er der Wiederkehr der erhabenen Schreibart bei Klopstock, der Idee, den großen Gedanken der Schöpfung noch einmal zu denken, die Goethes Naturstudium ins Empirische ausarbeitet. Der Enthusiasmus ist theologisch fundiert, mit dem Paracelsus das Sechstagewerk feiert und durch alle Reiche eindringlich befestigt:

also ist der mensch das fünfte wesen und ist microcosmus und ist der son der ganzen welt, aus ursachen das er durch die hant gottes also gemacht ist, ein auszug zu sein aus allen seinen geschöpfen. und wie ein kint vom vatter gelernet wird und vom vatter da ist, also lernen wir auch aus dem gestirn, daraus wir auch sein. darumb schlegt der mensch in die art der sternen, schlegt auch in die art der elementen, aus denen er dan gemacht ist. darumb er alle [ir] eigenschaft an im hat, darumb in auch die große welt speiset, füret und neret in weisheit, in vernunft, in speis und in trank, als sein eigen blut und fleisch, so wunderbarlich aus ir geboren. und das ist groß zu merken, das wir menschen essen und trinken unser fleisch und blut von unserm vatter der elementen und des firmaments. und darumb, das wir aus der welt gemacht, darumb speist uns die welt als ir kint. (Astronomia Magna oder die ganze Philosophia sagax der großen und kleinen Welt, 1537/38; S I, 12: 39f.) 